\title{
The dawn of a new era for dustless HdC stars with GAIA eDR3 *
}

\author{
P. Tisserand ${ }^{1}$, C.L. Crawford ${ }^{2}$, G.C. Clayton $^{2}$, A. J. Ruiter ${ }^{3}$, V. Karambelkar ${ }^{5}$, M.S. Bessell ${ }^{4}$, I. R. Seitenzahl ${ }^{3}$, \\ M. M. Kasliwal ${ }^{5}$, J. Soon ${ }^{4}$, T. Travouillon ${ }^{4}$,

\begin{abstract}
1 Sorbonne Universités, UPMC Univ Paris 6 et CNRS, UMR 7095, Institut d'Astrophysique de Paris, IAP, F-75014 Paris, France
2 Department of Physics \& Astronomy, Louisiana State University, Baton Rouge, LA 70803, USA

3 ARC Future Fellow, School of Physical, Environmental and Mathematical Sciences, University of New South Wales, Australian Defence Force Academy, Canberra, ACT 2600, Australia

${ }^{4}$ Research School of Astronomy and Astrophysics, Australian National University, Cotter Rd, Weston Creek ACT 2611, Australia

5 Cahill Center for Astrophysics, California Institute of Technology, Pasadena, CA 91125, USA
\end{abstract}

\begin{abstract}
Context. Decades after their discovery, only four hydrogen-deficient carbon (HdC) stars were known to have no circumstellar dust shell. This is in complete contrast to the $\sim 130$ known Galactic HdC stars that are notorious for being heavy dust producers, i.e. the $\mathrm{R}$ Coronae Borealis (RCB) stars. Together they form a rare class of supergiant stars that are thought to originate from the merger of $\mathrm{CO} / \mathrm{He}$ white dwarf (WD) binary systems, otherwise known as the double degenerate scenario.

Aims. We searched for new dustless $\mathrm{HdC}(\mathrm{dLHdC})$ stars to understand their Galactic distribution, to estimate their total number in the Milky Way and to study their evolutionary link with RCB stars and extreme Helium stars, the final phase of HdC stars.

Methods. We used primarily the 2MASS and GAIA eDR3 all-sky catalogues to select candidates that were then followed up spectroscopically. We studied the distribution of known and newly discovered stars in the HR diagram.

Results. We discovered 27 new dLHdC stars, one new RCB star, and two new EHe stars. Surprisingly, 20 of the new dLHdC stars share a characteristic of the known dLHdC star HD 148839, having lower atmospheric hydrogen deficiencies. The uncovered population of dLHdC stars exhibit a Bulge-like distribution, like the RCB stars, but show multiple differences from those that indicate they are a different population of $\mathrm{HdC}$ stars following its own evolutionary sequence with a fainter luminosity and also a narrow range of effective temperatures, between 5000 and $8000 \mathrm{~K}$. Not all the new dLHdC stars belong to that new population, as we found indication of a current low dust production activity around four of them: the warm F75, F152, C526 and the cold A166. Those could in fact be typical RCB stars passing through a transition time, going in or out of the RCB phase.

Conclusions. For the first time we have evidence of a large range of absolute magnitude in the overall population of HdC stars, spanning more than $3 \mathrm{mag}$. In the favoured formation framework, this is explained by a large range in the initial total WD binary mass which leads to a series of evolutionary sequences with distinct maximum brightness and initial temperature. The cold Galactic RCB stars are also noticeably fainter than the Magellanic ones, possibly due to a difference in metallicity between the original population of stars resulting in different WD mass ratio. The unveiled population of dLHdC stars indicates that the ability to create dust could be linked to the initial total mass. In our Galaxy, there could be as many dLHdC stars as RCB stars.
\end{abstract}

Key words. Stars: late-type - carbon - AGB and post-AGB - supergiants - circumstellar matter - Infrared:stars

\section{Introduction}

Hydrogen-deficient carbon (HdC) stars are a class of rare supergiant stars whose best known members are the R Coronae Borealis (RCB) stars (Clayton 1996, 2012; Tisserand et al. 2020). These latter are notorious for their surprising photometric variabilities as they undergo unpredictable large declines in brightness over a few weeks (up to 9 mag in the visible) before recovering their original brightness in a few months (see Tisserand et al. 2008, 2009, 2013 for some examples of light curves). These brightness variations are due to clouds of carbon dust ejected by the stars along the line of sight that obscure the photosphere. In doing so continuously, amorphous carbon dust grains (GarcíaHernández et al. 2011) build up around the stars and circumstellar dust shells form (Tisserand 2012, Montiel et al. 2015). Five members of the $\mathrm{HdC}$ stars formed a small subclass for decades as

Send offprint requests to: Patrick Tisserand; e-mail:
tisserand@iap.fr

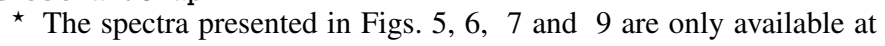
the CDS via anonymous ftp to cdsarc.u-strasbg.fr (130.79.128.5) or via http://cdsarc.u-strasbg.fr/viz-bin/cat/J/A+A/vol/page no variability was ever reported, despite the fact that they share the same peculiar spectroscopic characteristic with RCB stars (Warner 1967). Feast et al. (1997) called them the non-RCB HdC stars, but they were called simply HdC stars in many articles. They are HD 137613, HD 148839, HD 173409, HD 182040 and HD 175893. For the first four, Tisserand (2012) reported that no circumstellar dust shell has been detected with the WISE midinfrared space telescope (Wright et al.2010), supporting the fact that no carbon dust is currently being ejected from their atmospheres. From now on we will call them the dustless HdC (dL$\mathrm{HdC}$ ) stars to avoid any confusion with the HdC stars, their parent class. For the last one, HD 175893, it was found to possess a warm circumstellar dust shell, similar to that of RCB stars, indicating an on-going phase of dust production. This dust production did not coincide with a visual photometric decline. The reason is not known, it is possibly due to a particular location of the dust production site that prevent it from getting it on the line of sight.

Historically, HD 137613 and HD 173409 were reported nearly 130 years ago to be stars with peculiar spectra, respec- 
tively by Fleming (1892) and Pickering \& Fleming (1896), while HD 182040 was mentioned by Parkhurst (1912) to stand in a class by itself with a quote from Mrs Fleming writing "The lines in the spectrum are not those due to hydrogen (...)". Then Bidelman (1953) mentioned that the four following stars, HD 137613, HD 173409, HD 175893 and HD 182040 share similar spectra to the known variable RCB stars but did not show any brightness variations. He described spectra showing characteristic strong $\mathrm{C}_{2}$ band heads and some $\mathrm{C}$ I lines indicating an atmosphere rich in carbon, made mostly of isotope 12 as no evidence of the carbon isotope 13 was found. They are also hydrogen poor as no $\mathrm{CH}$ bands and no hydrogen absorption lines were detected. Ten years later, Warner (1963) reported a fifth star, HD 148839, that belongs to the group of hydrogen-poor carbon stars but with a hydrogen deficiency that is not so marked as the first four. Since then, only one dLHdC star has been reported. This is HE 10152050, a faint (G 16 mag) HdC star located in the Galactic Halo (Goswami et al. 2010). However, despite the fact that no IR excess has been observed from the 2MASS or WISE catalogues 1 . indicating that no warm circumstellar dust shell has built up in large quantity around that star, Goswami \& Aoki (2013) observed similar spectroscopic emission features that are usually seen in RCB stars that undergo photometric declines and inferred also the presence of circumstellar material from their polarimetric observations. These are really interesting observations as $\mathrm{HE}$ 1015-2050 could be useful to understand the evolutionary link between both types of HdC stars. Nevertheless, as no photometric variability has been observed in the past 16 years from the Catalina (Drake et al. 2012) and ASAS-SN (Shappee et al.|2014; Kochanek et al. 2017) monitoring surveys, we continue to classify HE 1015-2050 as a dLHdC star.

The distances and G-band brightnesses reported by the GAIA survey (Gaia Collaboration et al. 2016) for the first four dLHdC stars range respectively between 1 and $2 \mathrm{kpc}$, and between 6th and 9th magnitude. There was clearly room for progress in increasing their numbers, especially as an important successful effort has been made in the past 20 years to search for new RCB stars up to the distance of the Galactic Bulge (Zaniewski et al. 2005; Tisserand et al. 2008, 2011, 2013; Shields et al. |2019, Tisserand et al. 2020, Karambelkar et al. 2021) and in the Magellanic Clouds (Alcock et al. 1996, 2001; Tisserand et al. 2004, 2009). The search for new dLHdC stars is motivated to understand their Galactic distribution and to test the first estimate of their total Galactic numbers made by (Tisserand et al. 2020). Based only on the brightest 4 known dLHdC stars, it was estimated that there could exist up to one dLHdC star for every six RCB stars. Clearly this first estimate has a large uncertainty due to the small initial sample. It is important to evaluate this ratio accurately as it is used to estimate the total number of Galactic HdC stars, giving a strong constraint on their formation rate. As $\mathrm{HdC}$ stars are thought to result from the merger of one CO- plus one He- WD (Webbink 1984, Jeffery et al. 2011; Clayton 2012), such constraints are used to test the double degenerate scenario when compared to the birthrate of such mergers obtained by theoretical population synthesis, that is between $\sim 10^{-3}$ and $\sim 5 \times 10^{-3}$ per year (Nelemans et al. 2001, Ruiter et al. 2009; Karakas et al. 2015).

Finally we invite the readers to read the complementary analysis made by Crawford et al. (2022) and Karambelkar et al.

\footnotetext{
1 2MASS: $(\mathrm{J}-\mathrm{H})_{0} \sim 0.20 \mathrm{mag}$ and $(\mathrm{H}-\mathrm{K})_{0} \sim 0.27 \mathrm{mag}$, WISE: [3.4][4.6] 0 mag, only magnitude limit values were reported for the reddest WISE bands, [12] and [22], in both WISE All-Sky and ALLWISE catalogues
}

(2022) that were respectively carried out on the optical and near IR spectra of some of these new dLHdC stars. The first paper looks at the astonishing strong strontium and barium absorption lines we found in A249, C539 and A166 and uses stellar evolution models to estimate the type of neutron exposure that occurs in a typical HdC star to explain the observed enhanced s-process material on their surfaces. The second analysis measured the oxygen isotopic ratio ${ }^{16} \mathrm{O} /{ }^{18} \mathrm{O}$ of all new cold $\mathrm{dLHdC}$ stars for which the $\mathrm{CO}$ band heads were detectable in the $\mathrm{K}$ band and compared them to values obtained for RCB stars. They confirmed the lower ${ }^{16} \mathrm{O} /{ }^{18} \mathrm{O}$ ratio observed in dLHdC stars as indicated by Clayton et al. (2007) and García-Hernández et al. (2009) who suggested that dLHdC stars have ${ }^{10} \mathrm{O} /{ }^{18} \mathrm{O}<1$ and most RCB stars have ${ }^{16} \mathrm{O} /{ }^{18} \mathrm{O}>1$.

We searched for new dLHdC stars using the datasets of two all-sky surveys: 2MASS (Skrutskie et al.2006) and GAIA eDR3 (Gaia Collaboration et al. 2021). We limit ourselves to stars whose lines of sight are affected by an integrated interstellar reddening $\mathrm{E}(\mathrm{B}-\mathrm{V})<1 \mathrm{mag}$ (Schlafly \& Finkbeiner 2011). The selection of candidates based on their brightness and colour is detailed in section 2, then in section 3 we describe the spectroscopic follow-up of these candidates and the subsequent discovery of new dLHdC, RCB and EHe stars. In section 4 we discuss the properties of this population of dLHdC stars and compare them to the known Galactic RCB stars, on spatial distribution, brightness, effective temperature, photometric variability, dust production rate and spectral energy distribution. We also estimate their total number in the Milky Way, before summarising our results in section 5

\section{Analysis}

\subsection{Initial star lists}

With the release of the GAIA eDR3 all-sky catalogue, a large scale search of rare objects such as dLHdC stars is now possible as supergiant stars can be selected up to the distance of the Galactic Bulge thanks to the precise parallax measurements reported. Then, as an initial selection, we downloaded all giant stars with a Gaia $\mathrm{M}_{G}<0$ mag, Bp-Rp $>0.5$ mag, an apparent $G$ magnitude brighter than $15^{\text {th }}$ mag and an estimated parallax SNR (signal-to-noise ratio) higher than 3. We used here, for simplification, the inverse of the GAIA eDR3 parallax as a first distance estimate. The catalogue was simply cleaned of possible wrong parallax measurements using the two following constraints on GAIA eDR3 parameters, RUWE $\leq 1.4$ or ipd_gof_harmonic_amplitude $\leq 0.1$, as recommended by Lindegren et al. (2021b). HE 1015-2050, being fainter than $15^{t h}$ magnitude and with no significant parallax measurement reported by GAIA eDR3, is the only known dLHdC star that did not fully pass these initial criteria.

Next, we cross-matched this sub-sample of GAIA stars with the ALLWISE catalogue (Cutri R.M. 2013) using the GAIA archive service (https://gea.esac.esa.int/archive/). This catalogue contains the WISE mid-infrared magnitudes but also the 2MASS $\mathrm{J}, \mathrm{H}$, and $\mathrm{K}$ near-IR magnitudes as an internal cross-matching was already performed. However, not all GAIA stars were matched to an ALLWISE counterpart, therefore we also crossmatched with a $1^{\prime \prime}$ radius association criterion those remaining GAIA stars to the 2MASS catalogue using the CDS crossmatching tools (http://cdsxmatch.u-strasbg.fr/). This was the case for the known dLHdC star, HD 182040. We also calculated for all selected GAIA stars an E(B-V) interstellar reddening magnitude following Schlafly \& Finkbeiner (2011). The GAIA 

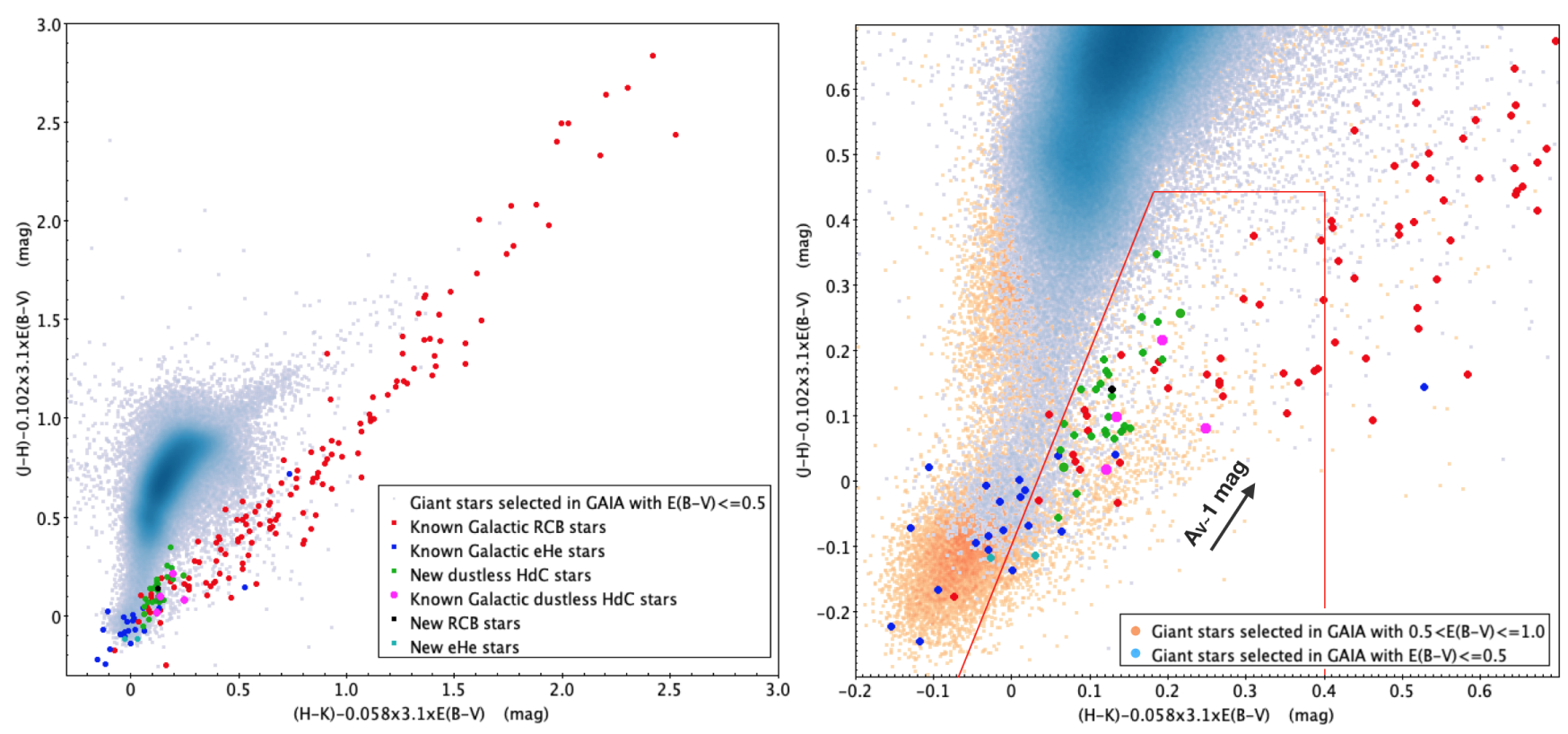

Fig. 1: Colour-colour $(\mathrm{J}-\mathrm{H})_{0}$ vs $(\mathrm{H}-\mathrm{K})_{0}$ diagram with 2MASS magnitudes corrected from extinction showing the positions of all known and new Galactic HdC and EHe stars, represented with colour points, in comparison to giant stars $\left(\mathrm{M}_{G} \leq 0 \mathrm{mag}, \mathrm{Bp}-\mathrm{Rp}>0.5\right.$ mag, $\mathrm{G}<15 \mathrm{mag}$ ) selected in GAIA eDR3 (Groups A and B). Left: distribution of all giant stars with an interstellar reddening E(BV) lower than $0.5 \mathrm{mag}$. Right: zoom on the lower left corner. The distribution of giant stars impacted with an interstellar reddening between $0.5<\mathrm{E}(\mathrm{B}-\mathrm{V}) \leq 1.0 \mathrm{mag}$ are indicated in red, while the others are in blue. The selection area is delimited with red lines. The interstellar dust reddening vector is represented for an extinction $\mathrm{A}_{V}$ of 1 mag.

Bp-Rp colour and $\mathrm{G}$ brightness were corrected using these simple formula: $\triangle(\mathrm{Bp}-\mathrm{Rp}) \sim 2 \times \mathrm{E}(\mathrm{B}-\mathrm{V})$ for the reddening correction and $\mathrm{A}_{G} \sim 3.1 \times \mathrm{E}(\mathrm{B}-\mathrm{V})$ for the visual extinction (Andrae et al. 2018; Malhan et al. 2018). For simplicity, we kept our search for new dLHdC stars in the sky area impacted by an interstellar reddening $\mathrm{E}(\mathrm{B}-\mathrm{V})$ lower than 1 magnitude.

For the definition of the subsequent selection criteria on absolute brightness and colour, we categorised our initial samples of GAIA stars detailed above in four groups. Groups A and C contain the giant stars that have a successful match with the WISE ALLWISE catalogue, but were reported with a parallax SNR respectively higher than 5, and between 3 and 5. Groups $\mathrm{B}$ and $\mathrm{D}$ were defined with the same split on parallax but only with the stars for which no association with the WISE ALLWISE catalogue was found and a cross-matching with the 2MASS catalogue was then necessary.

Two more groups, Group F and G, were defined to open up our search to GAIA stars reported with no significant distance estimate (parallax SNR lower than 3) towards sky areas impacted by an interstellar reddening $\mathrm{E}(\mathrm{B}-\mathrm{V})<0.5 \mathrm{mag}$. This initial set of stars is also following the criteria defined above on the GAIA $G$ apparent brightness $(\mathrm{G}<15 \mathrm{mag}$ ) and colour (Bp-Rp $>0.5 \mathrm{mag})$. Similarly to the first four groups, Group F corresponds to stars with a WISE ALLWISE catalogue counterpart, while stars from Group G have only a 2MASS association.

Overall we started our analysis with an initial set of about 3.5 millions GAIA stars, $\sim 85 \%$ of them being located on lines of sight with median interstellar dust reddenings lower than E(B$\mathrm{V}) \leqslant 0.5 \mathrm{mag}$ (see Table 1 for the details on the distribution of these stars among the different groups).

\subsection{Selection criteria}

We used primarily the GAIA eDR3 and the 2MASS all-sky catalogues to select targets that we followed up spectroscopically. The mid-IR WISE magnitudes were used to verify that no large fraction of selected stars belonging to Groups A, C, and F possessed a clear circumstellar dust shell. Indeed, we found that a large majority of the stars that passed our first two primary selection cuts, detailed below, have their [4] - [12] and [12] - [24] colour indices distributed around zero. Therefore we did not apply strict criteria on their WISE colours to retain potential discoveries of new RCB stars that usually show an excess due to warm dust surrounding them. After the initial selection of giant stars from the GAIA eDR3 catalogue, we applied the three selection criteria described below. The overall selection process is summarised in Table 1 for Groups A to D.

First, we selected a first sample of stars whose position in the $(\mathrm{J}-\mathrm{H})_{0}$ vs $(\mathrm{H}-\mathrm{K})_{0}$ colour-colour diagram, corrected for interstellar reddening, is similar to the four brightest known dLHdC stars. The area selected corresponds to stars with no high IR excess unlike most RCB stars. Fortuitously, the four known dLHdC stars are also separated from the main locus of classical giant stars with a redder H-K colour index. We therefore defined some specific limits that are illustrated in Figure 1. This selection has a high impact as it rejected about $99.7 \%$ of the initial sample of giant stars. In practice, we selected stars using the following criteria with $\alpha=0.1$ :

$$
\begin{aligned}
& \text { if } 0.18<(H-K)_{0}<0.4:(J-K)_{0}<0.44 \\
& \text { if }(H-K)_{0} \leqslant 0.18:(J-H)_{0}<3 \times(H-K)_{0}-\alpha
\end{aligned}
$$

Second, we used the GAIA eDR3 $\mathrm{M}_{G}$ vs (Bp-Rp) $)_{0}$ colour magnitude diagram to select stars whose brightness and colour 

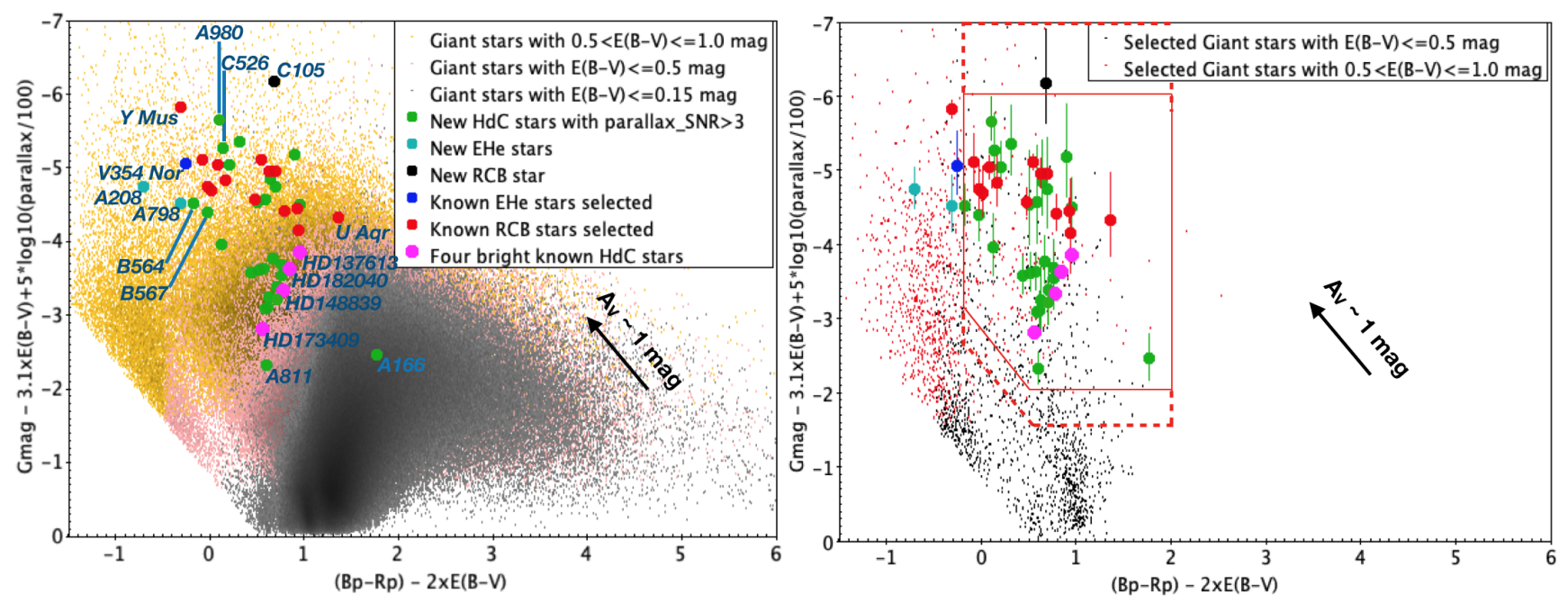

Fig. 2: HR diagram $\mathrm{M}_{G}$ vs (Bp-Rp) with GAIA magnitudes corrected for the interstellar reddening. Left: distribution of all giant stars whose measured parallax SNR is higher than 5 (Group A and B) but impacted by a low level of interstellar reddening $(\mathrm{E}(\mathrm{B}-\mathrm{V}) \leq$ $0.15 \mathrm{mag}$, grey dots), a medium one $(\mathrm{E}(\mathrm{B}-\mathrm{V}) \leq 0.5 \mathrm{mag}$, pink dots $)$ or an even higher level $(0.5<\mathrm{E}(\mathrm{B}-\mathrm{V}) \leq 1.0 \mathrm{mag}$, orange dots $)$. The position of the known HdC and EHe stars that have passed the first selection cuts are indicated, as well as the newly discovered ones. Right: Same as left, except that the giant stars represented are the ones that have passed the first selection cuts. The black dots correspond to the objects impacted by a medium value of interstellar reddening, $E(B-V) \leq 0.5$ mag, while the red ones are impacted by higher values up to $1.0 \mathrm{mag}$. The error bars on the y axes due to the parallax measurement accuracy are represented only on the right diagram for clarity. The selection areas are illustrated with red lines: solid ones for groups A and B, and dashed ones for groups C and D whose parallax is measured with an SNR lower than 5. The interstellar extinction of $\mathrm{A}_{V} \sim 1$ mag is represented with a black arrow.

were similar to the 4 known dLHdC stars. The selection area is illustrated in Figure 2, right side. As we have chosen, for simplicity, to use the inverse of the GAIA eDR3 parallax as a distance estimate, we loosen up our cuts on the brightness scale. A more accurate geometric distance estimate inferred by BailerJones et al. (2021) is used is Sect.4.2 to study in details the population of dLHdC stars. Overall, the limits were chosen pragmatically, with the goal to keep deliberately the range of colour and brightness as wide as possible while maximizing the rejection of two group of stars: the bright blue clump of stars and the group of fainter giant stars whose colour (Bp-Rp 1.0 mag) is similar to the stars of the Red Clump which are one magnitude fainter (see Gaia Collaboration et al.2018, Fig.10). The selection limits were chosen to be even wider on the brightness scale for Groups $\mathrm{C}$ and $\mathrm{D}$ due to the higher imprecision on the knowledge of their respective distances. About $12 \%$ of the stars that have passed cut \#1 were selected. It is interesting to note that the four known $\mathrm{dLHdC}$ stars, used as reference in our analysis, lie in a position of the HR diagram which is underpopulated when we compare them to some giant stars impacted by a very low interstellar reddening, i.e. $\mathrm{E}(\mathrm{B}-\mathrm{V}) \leq 0.15 \mathrm{mag}$ (see Figure 2, left side). The 4 known dLHdC stars are brighter and bluer than the main locus of stars formed mainly by the asymptotic giant branch (AGB) stars. In sky areas of higher interstellar reddening, the imprecision on the knowledge of the interstellar extinction has the direct consequence of dispersing the distribution of stars in the HR diagram. We can therefore expect that most of the selected stars will be AGB stars but some hot blue stars whose extinction correction was underestimated will also be selected.

Finally for the third and last selection, we queried the CDS/SIMBAD database (Wenger et al. 2000) for information on all stars that pass the selection cuts described above. We then rejected all targets that possess already a well defined spectro- scopic type that is not carbon rich, and all targets associated with a globular cluster. Obviously we removed also all known dLHdC stars and RCB stars that have passed through all the applied criteria. There were 18 of them which are listed in Table 1. About $38 \%$ of the stars selected in cut \#2 were removed at this stage from the final target list. We selected 631 stars from Groups A to D for spectroscopic follow-up, with $\sim 44 \%$ of them being located in sky area of low interstellar reddening, i.e. $\mathrm{E}(\mathrm{B}-\mathrm{V}) \leqslant 0.5 \mathrm{mag}$. As we started with an initial dataset whose such stars represented $\sim 85 \%$ of the total, our selection process was less efficient with stars impacted by higher extinction.

For the nearly 400 thousand stars belonging to Groups $F$ and $\mathrm{G}$, whose measured parallax SNR is lower than 3, we applied a stricter selection on their 2MASS near-IR colours with $\alpha=0.2$ in Eq. 1 because of a higher dispersion. We also requested a tighter constraint on their GAIA $\left(\mathrm{B}_{p}-\mathrm{R}_{p}\right)_{0}$ colour, ranging between 0.4 and $1.2 \mathrm{mag}$ after interstellar dust correction, and on their GAIA $G$ apparent brightness with $G<13.5$ mag. Then, for Group F only, we added a final cut to reject stars that presented magnitude limits in the [12] or [24] WISE photometric bands. After a cross match with the SIMBAD database, we selected 89 supplementary targets, after removing two known RCB stars: ASAS J050232-7218.9 and UX Ant.

\section{Discoveries}

\subsection{Spectroscopic follow-up}

The spectroscopic follow-up of all targets was conducted with the Wide Field Spectrograph (WiFeS) instrument (Dopita et al. 2007) attached to the $2.3 \mathrm{~m}$ telescope of the Australian National University at Siding Spring Observatory (SSO). WiFeS is an integral-field spectrograph permanently mounted at the Nasmyth 
Table 1: Number of selected Galactic objects after each selection criterion.

\begin{tabular}{|c|c|c|c|c|c|c|}
\hline \multirow{4}{*}{$\begin{array}{l}\text { Selection } \\
\text { criterion }\end{array}$} & \multicolumn{4}{|c|}{ Groups } & \multirow[t]{4}{*}{ Total } & \multirow{4}{*}{$\begin{array}{c}\text { Fraction of stars with } \\
E(B-V) \leqslant 0.5\end{array}$} \\
\hline & \multirow{2}{*}{\multicolumn{2}{|c|}{$\begin{array}{c}\text { A } \\
\text { Parallax SNR } \geq 5\end{array}$}} & \multirow{2}{*}{\multicolumn{2}{|c|}{$\underset{3 \leq \text { Parallax }}{\text { CNR }}<$ D }} & & \\
\hline & & & & & & \\
\hline & ALLWISE & 2MASS & ALLWISE & 2MASS & & \\
\hline Cut \#0 (initial set) & 1484627 & 1007019 & 509965 & 122681 & 3124292 & $\sim 85 \%$ \\
\hline Cut \#1 & 3218 & 891 & 720 & 488 & 5317 & $\sim 67 \%$ \\
\hline Cut \#2 & 552 & 162 & 132 & 170 & 1016 & $\sim 48 \%$ \\
\hline Cut \#3 & 299 & 78 & 118 & 154 & 649 & $\sim 45 \%$ \\
\hline $\begin{array}{l}\text { Known dLHdC/RCB } \\
\text { stars removed }\end{array}$ & $12^{\star A}$ & $4^{\star B}$ & $1 \star C$ & $1 \star D$ & 18 & $\sim 55 \%$ \\
\hline Total selected & 287 & 74 & 117 & 154 & 631 & $\sim 44 \%$ \\
\hline Observed & 139 & 43 & 84 & 91 & 357 & $\sim 65 \%$ \\
\hline $\begin{array}{l}\text { New dLHdC/RCB } \\
\text { stars found }\end{array}$ & 11 & 6 & 9 & 0 & 26 & $\sim 46 \%$ \\
\hline \multicolumn{7}{|c|}{$\begin{array}{l}{ }^{\star A}: \text { HD 137613, HD 173409, HD 148839, HD 175893, V2552 Oph, V532 Oph, ASAS-RCB-10, } \\
\text { ASAS-RCB-3, ASAS-RCB-8,GU Sgr, WISE_J172447.52-290418.6 and WISE_J172951.80-101715.9 } \\
{ }^{\star B}: \text { HD 182040, RT Nor, RY Sgr and SV Sge } ;{ }^{\star C}: \text { WISE_J182943.83-190246.2; }{ }^{\star D}: \text { U Aqr }\end{array}$} \\
\hline
\end{tabular}

A focus. It provides a $25^{\prime \prime} \times 38^{\prime \prime}$ field of view with $0.5^{\prime \prime}$ sampling along each of the twenty-five $38^{\prime \prime} \times 1^{\prime \prime}$ slitlets. The visible wavelength interval is divided by a dichroic at around $600 \mathrm{~nm}$, feeding two essentially similar spectrographs. The spectra have a twopixel resolution of $2 \AA$ and wide wavelength coverage, from 340 to $960 \mathrm{~nm}$. We observed 427 targets with WiFeS during 21 nights between April and October 2021. The spectroscopic dataset collected was reduced using the PyWiFes data reduction pipeline (Childress et al.2014).

The sky distribution of all targets selected for spectroscopic follow-up is presented in Figure 3. Nearly $\sim 60 \%$ of all selected targets were observed, which included almost all targets located within 45 degrees from the Galactic centre. There, we had almost no available targets located within 3 degrees from the Galactic plane. Among the targets we did not observe, $\sim 78 \%$ of those (i.e., $31 \%$ of total) are located in a sky area which could not be reached with the telescope during our 7 months long observing campaign, i.e. Dec $>+25$ degrees and/or $6<\mathrm{RA}<10 \mathrm{H}$. All the remaining ones were considered as low priority targets because at least one magnitude limit was reported in one of the three photometric 2MASS IR bands. Most of the time, this indicated that the source was strongly blended with a nearby object.

After inspection of the spectra collected, we found 27 new dustless HdC stars, one new RCB star and 2 extreme Helium (EHe) stars. They are listed in Table 2 with the names used in the study, their GAIA eDR3 parallax SNR and magnitudes, and their estimated geometric distance with a 1 sigma error (BailerJones et al. 2021). We'll first discuss the new HdC stars and then provide details on the two new EHe stars in section 3.3 .

\subsection{New $d L H d C$ and RCB stars}

The spectra of all new HdC stars stand out. We looked principally for an absence or weakness of any hydrogen Balmer lines and the absence of the $\mathrm{CH}$ band head located at around $\sim 4300 \AA$. Then, we compared individually the selected spectra to the ones of the hundred known HdC stars we collected so far to recognise the typical metallic and carbon absorption patterns in relation to the effective temperature, i.e. the $\mathrm{C} \mathrm{I}$ absorption lines for the warm HdC stars $\left(\mathrm{T}_{\text {eff }}>6500 \mathrm{~K}\right)$ and the $\mathrm{CN}$ and $\mathrm{C}_{2}$ band heads observed for the colder ones. New HdCs were required to have spectra closely resembling at least one of the known HdC stars

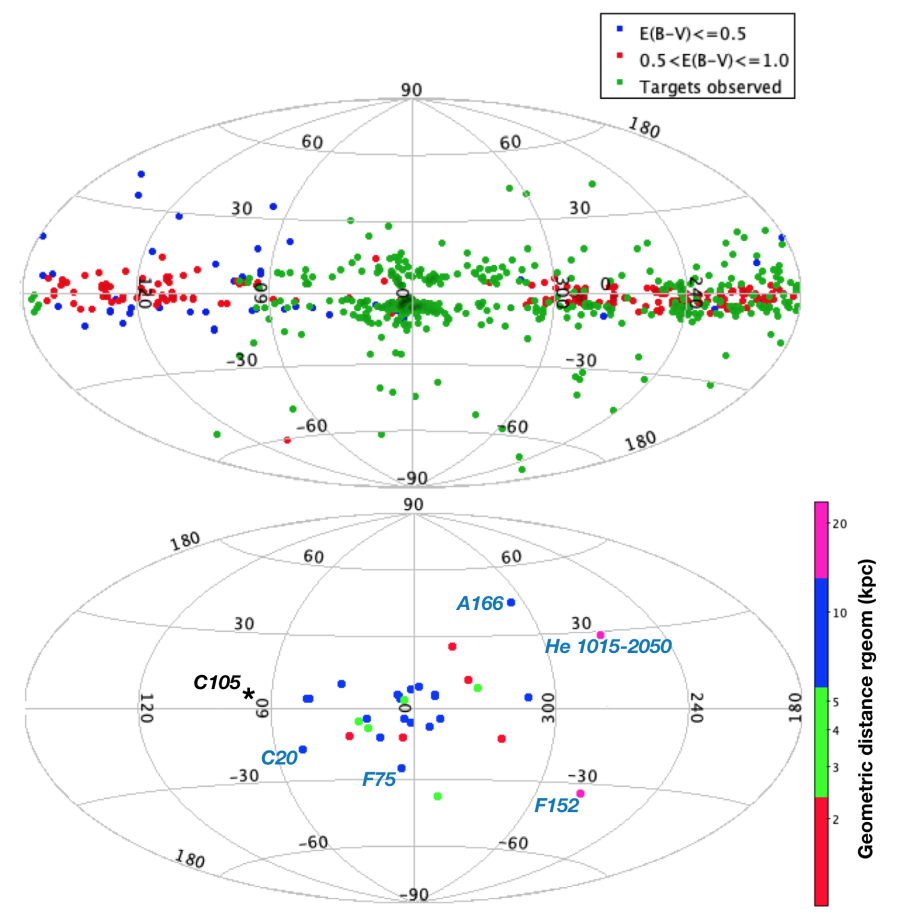

Fig. 3: Spatial distribution in Galactic coordinates. Top: all selected targets for spectroscopic follow-up with the targets already observed in green, the remaining ones are shown in blue if under an interstellar reddening lower than $\mathrm{E}(\mathrm{B}-\mathrm{V}) \leqslant 0.5 \mathrm{mag}$ and red for higher reddening, up to $1.0 \mathrm{mag}$. Bottom: all dLHdC stars colour coded with their respective geometric distances rgeom inferred by Bailer-Jones et al. (2021). The names of all dLHdC stars that are most certainly located in the Galactic Halo have been indicated. The location of the new RCB star, C105 is indicated with a black star.

in order to be confirmed as members of the class. Detailed discussions on the characteristics of these spectra and some presentations of them can be found in Tisserand et al. (2020). Additionally, we note that 6 of the new dLHdC stars (A182, A183, A223, B42, C20 and C27) were already catalogued as carbon rich stars in the General catalogue of carbon stars (Alksnis et al. 2001). A 
Table 2: New HdC and EHe stars discovered

\begin{tabular}{|c|c|c|c|c|c|c|c|c|c|}
\hline Star & $\begin{array}{c}\text { 2MASS } \\
\text { Id }\end{array}$ & $\begin{array}{l}\text { Other } \\
\text { names }\end{array}$ & RA & Dec & $\begin{array}{c}\text { GAIA } \\
\text { parallax } \\
\text { SNR }\end{array}$ & $\begin{array}{l}\text { Distance }^{\star} \\
(\mathrm{kpc}) \text { with } \\
1 \text { sig error }\end{array}$ & $\begin{array}{c}\text { GAIA } \\
\text { G } \\
\text { mag }\end{array}$ & $\begin{array}{c}\text { GAIA } \\
\text { Bp-Rp } \\
\text { mag }\end{array}$ & $\begin{array}{c}\mathrm{E}(\mathrm{B}-\mathrm{V})^{\diamond} \\
\mathrm{mag}\end{array}$ \\
\hline
\end{tabular}

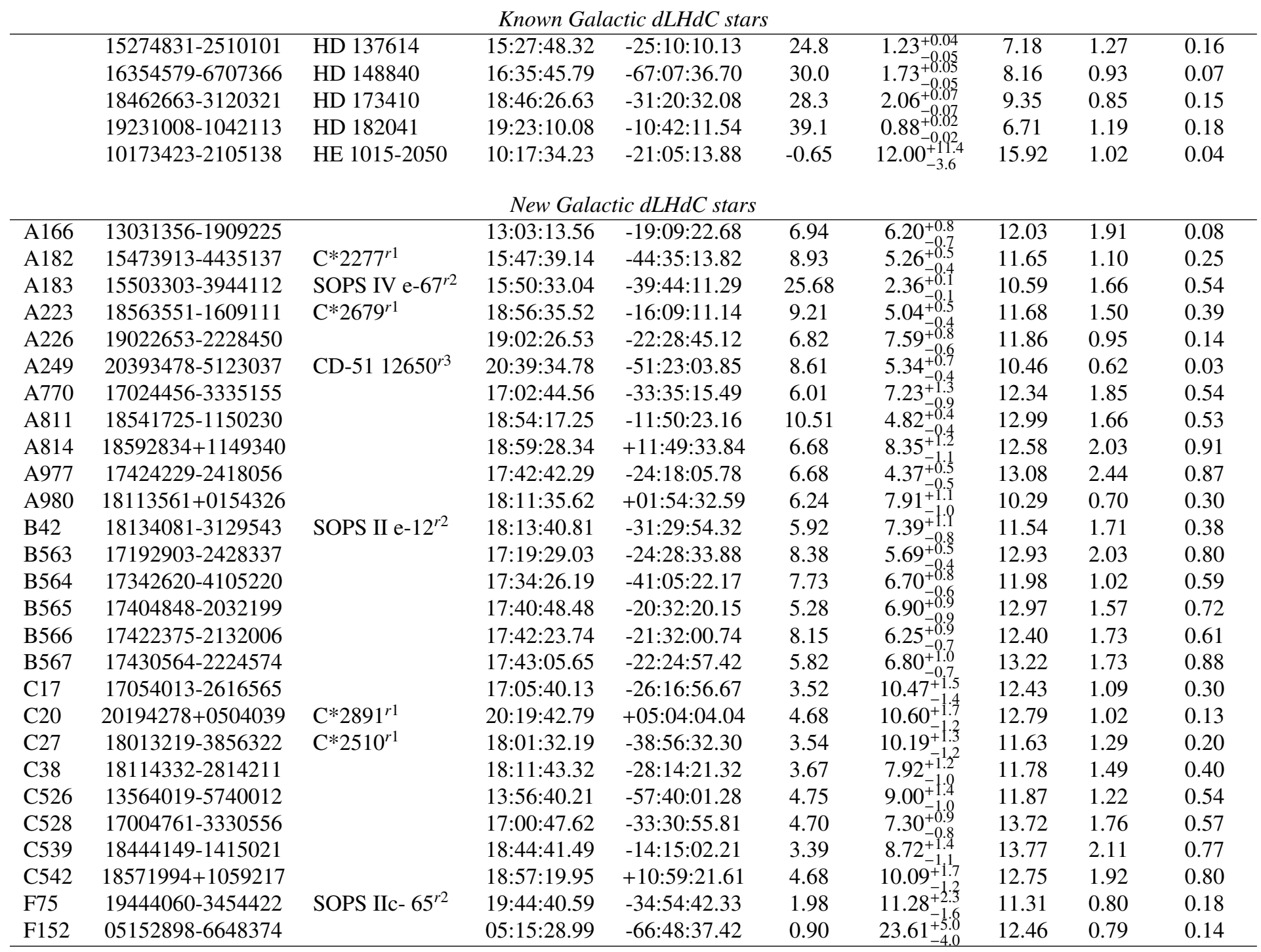

New Galactic RCB star

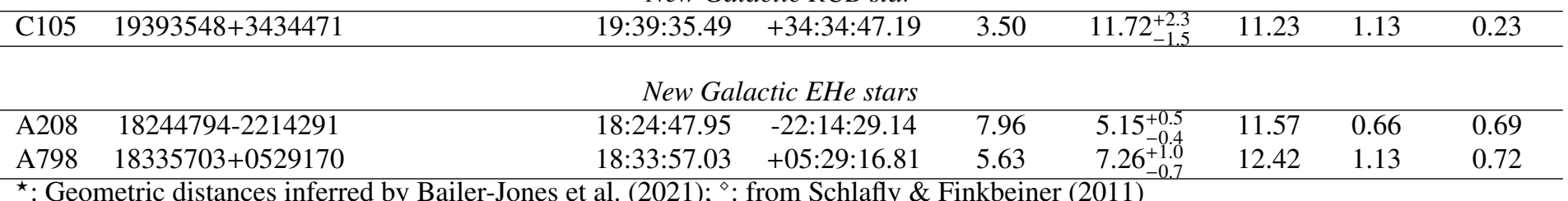

*: Geometric distances inferred by Bailer-Jones et al. (2021); ${ }^{\circ}$ from Schlafly \& Finkbeiner (2011)

${ }^{r 1}:$ Stephenson (1973); Alksnis et al. (2001); ${ }^{r 2}:$ Stock \& Wroblewski $(1972) ;{ }^{r 3}$ : The "Cordoba Durchmusterung" catalogue (1932)

spectral classification of type R ( 2 for A182) was given by the $\mathrm{CDS} / \mathrm{SIMBAD}$ database indicating they are warmer than classical carbon stars.

One first marked difference we observed is the general weakness of the $\mathrm{CN}$ bands in the new dLHdC stars compared to known RCB stars. For a new dLHdC star and a known RCB star with similar $C_{2}$ band strengths, and thus presumably similar temperatures, the dLHdC stars generally show significantly weaker $\mathrm{CN}$ bands. Assuming the similarities in $\mathrm{C}_{2}$ band strength of these two stars indicates a similar carbon abundance, the weakness of the $\mathrm{CN}$ bands implies that the new dLHdC stars have a lower nitrogen abundance. More details on this comparison will be made available by Crawford et al. (2022, in prep.). However we note that such lower $\mathrm{N}$ abundance was not detected by GarcíaHernández et al. (2009) high-resolution IR spectroscopic analysis. They observed three of the known dLHdC stars and three RCB stars (HD 175893, S Aps and Y Mus), and reported similar high $\mathrm{N}$ abundances for all. But nonetheless, we should be 


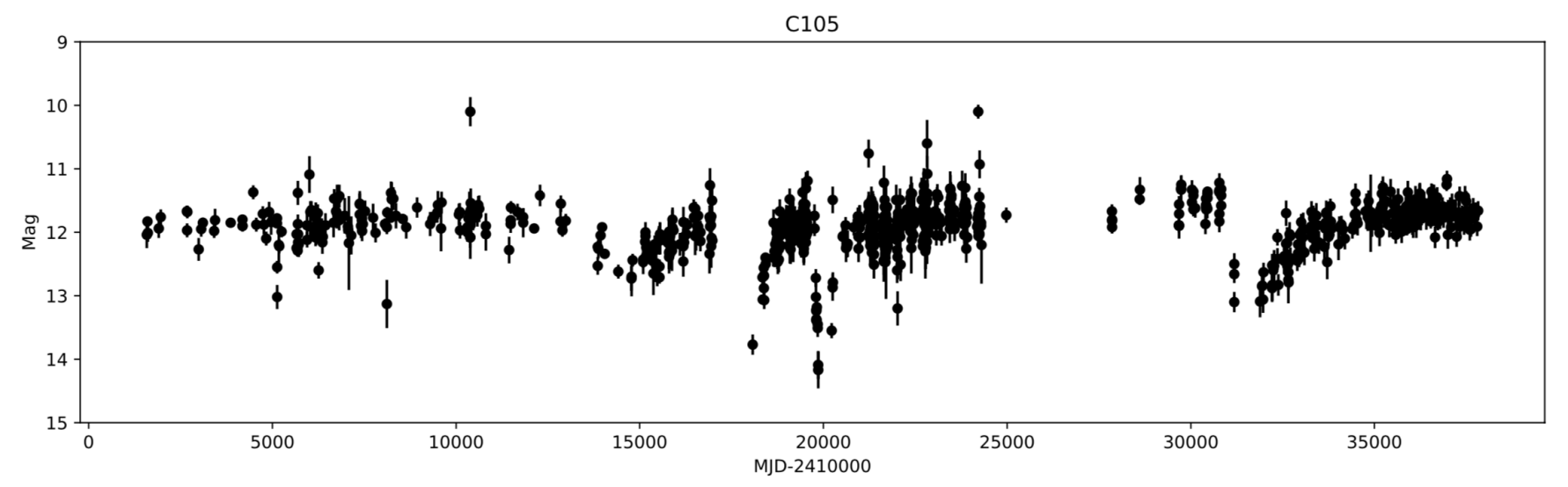

Fig. 4: DASCH light curve of C105 covering about 100 years from $\sim 1890$ to $\sim 1990$. The average plate limit is $\sim 13.5$ mag.

Table 3: Spectroscopic and photometric characteristic observed in new and Known HdC stars

\begin{tabular}{|c|c|c|c|c|c|}
\hline \multirow[t]{2}{*}{ stars } & \multicolumn{2}{|c|}{ Line strength } & \multirow{2}{*}{$\begin{array}{l}\text { Temp. } \\
\text { group }\end{array}$} & \multirow{2}{*}{$\begin{array}{c}\mathrm{M}_{V} \\
(\mathrm{mag})\end{array}$} & \multirow{2}{*}{$\begin{array}{l}(\mathrm{V}-\mathrm{I})_{0} \\
(\mathrm{mag})\end{array}$} \\
\hline & $\mathrm{H}$ & $\mathrm{Li}$ & & & \\
\hline \multicolumn{6}{|c|}{$d L H d C$ stars } \\
\hline HD 137613 & & & Mild & $-3.29_{-0.08}^{+0.09}$ & 1.17 \\
\hline HD 148839 & ++ & + & Mild & $-3.09_{-0.07}^{+0.08}$ & 0.66 \\
\hline HD 173409 & & & Mild & $-2.47_{-0.07}^{+0.04}$ & 0.92 \\
\hline HD 182040 & & & Cold & $\begin{array}{r}-0.07 \\
-3.28^{+0.05}\end{array}$ & 1.01 \\
\hline HE $1015-2050$ & & & Cold & $\mathrm{nd}^{-0.05}$ & 0.88 \\
\hline A166 & & & Cold & $-1.38^{+0.27}$ & 1.80 \\
\hline A182 & & & Warm & $\begin{array}{l}-2.46_{-0.20}^{+0.19} \\
-0.04\end{array}$ & $0.63^{\triangle}$ \\
\hline A183 & ++ & + & Mild & $\begin{array}{l}-2.37^{+0.007} \\
-0.09\end{array}$ & 0.84 \\
\hline A223 & + & & Cold & $\begin{array}{r}-0.09 \\
-2.96^{+0.16}\end{array}$ & 0.70 \\
\hline A 226 & & & Mild & $\begin{array}{r}-0.19 \\
-2.85_{-020}^{+0.19}\end{array}$ & 0.60 \\
\hline A249 & ++ & & Warm & $-3.22_{-0.26}^{+0.18}$ & 0.45 \\
\hline A770 & + & & Cold & $-2.87^{+0.27}$ & 1.10 \\
\hline A811 & ++ & + & Cold & $\begin{array}{r}-0.36 \\
-2.05_{-018}^{+0.18}\end{array}$ & 0.62 \\
\hline A814 & ++ & & Mild & $-4.26_{-0.0}^{+0.18}$ & 0.64 \\
\hline A977 & & & Cold & $\begin{array}{r}-0.29 \\
-2.75_{-0.2}^{+0.27}\end{array}$ & 0.73 \\
\hline A980 & + & + & Warm & $-5.18_{-0.29}^{+0.28}$ & $0.16^{\nabla}$ \\
\hline B42 & + & & Mild & $\begin{array}{r}-0.29 \\
-3.39^{+0.24}\end{array}$ & 0.96 \\
\hline B563 & + & ++ & Mild & $\begin{array}{r}-0.29 \\
-2.96_{-020}^{+0.18}\end{array}$ & 0.75 \\
\hline B564 & + & + & Warm & $\begin{array}{r}-3.75_{-0.04}^{+0.10} \\
-\end{array}$ & $0.11^{\nabla}$ \\
\hline B565 & ++ & + & Mild & $\begin{array}{r}-2.97_{-0.30}^{+0.24} \\
-0.26\end{array}$ & 0.57 \\
\hline B566 & & & Mild & $\begin{array}{r}-0.26 \\
-3.07_{-0.28}^{+0.25}\end{array}$ & 0.78 \\
\hline B567 & & & Warm & $\begin{array}{r}-3.33_{-0.30}^{+0.25} \\
-\end{array}$ & 0.27 \\
\hline $\mathrm{C} 17$ & ++ & + & Mild & $\begin{array}{r}-3.55_{-0.9}^{+0.30} \\
-\end{array}$ & $0.47^{\nabla}$ \\
\hline $\mathrm{C} 20$ & + & + & Mild & $\begin{array}{r}-2.41_{-0.26}^{+0.26} \\
\end{array}$ & 0.82 \\
\hline $\mathrm{C} 27$ & & & Cold & $-3.65_{-0.26}^{+0.27}$ & 0.85 \\
\hline $\mathrm{C} 38$ & + & + & Mild & $-3.71_{-0.30}^{+0.36}$ & 0.76 \\
\hline C526 & + & & Warm & $-4.29_{-0.31}^{+0.26}$ & 0.35 \\
\hline C528 & ++ & + & Mild & $-1.81_{-0.24}^{+0.26}$ & 0.81 \\
\hline C539 & & & Mild & $-2.98^{+0.31}$ & 0.84 \\
\hline C542 & ++ & + & Mild & $-4.14_{-0.34}^{+0.29}$ & 0.70 \\
\hline F75 & + & + & Warm & $-4.41_{-0.40}^{+0.34}$ & 0.39 \\
\hline F152 & Em. & & Warm & $\begin{array}{r}-0.40 \\
-4.76_{-042}^{+0.40}\end{array}$ & 0.47 \\
\hline \multicolumn{6}{|c|}{ RCB stars } \\
\hline C105 & & & Mild & $-4.64^{+0.29}$ & 0.61 \\
\hline HD 175893 & & & Mild & $-3.99_{-0.15}^{+0.15}$ & 0.61 \\
\hline
\end{tabular}

${ }^{\triangle}$ Interstellar reddening correction possibly underestimated

${ }^{\nabla}$ Interstellar reddening correction possibly overestimated cautious with their result as they commented that their derived $\mathrm{N}$ abundances for the dLHdC star, HD 137613, and the RCB star, Y Mus, are in poor agreement (difference of $\sim 0.7$ dex) with other analysis made respectively by Kipper (2002) and Asplund et al. (2000) based on high resolution visible spectroscopy, and that the issue was not properly investigated. Since in RCB stars, the abundance of most metals, like $\mathrm{N}$, are roughly proportional to the one of Fe (Jeffery et al. 2011), we can infer that the metallicity of dLHdC stars is probably lower than most HdC stars. This will need future investigations for confirmation.

From a careful spectral comparison with known HdC stars and models, we attributed also a temperature to all new dLHdC stars, from the coldest, A166 ( $\mathrm{T}_{\text {eff }} \sim 4000 \mathrm{~K}$ ), to the warmest, B564 ( $\left.\mathrm{T}_{\text {eff }} \sim 8000 \mathrm{~K}\right)$. A detailed study on this work and for the hundreds known RCB stars can be found in (Crawford et al. 2022, in prep.). We then classified them in the three following groups depending of a temperature range: Cold for $\mathrm{T}_{e f f} \leq 5750 \mathrm{~K}$, Mild for $5750<\mathrm{T}_{\text {eff }} \leq 6500 \mathrm{~K}$ and Warm for $6500<\mathrm{T}_{\text {eff }} \leq 8000 \mathrm{~K}$. Those are listed in Table 3 and their spectra are presented respectively in Figures 5- 7] We found that the majority of the new dLHdC stars (13) have a temperature similar to the 3 historically known ones and belong to the Mild group, while 6 are considered as Cold but with a temperature near 5500 $\mathrm{K}$ for 5 of them and the remaining 8 are classified as Warm. We were surprised to discover such warm dLHdC stars as none were known before and our analysis did not target them specifically. Their temperatures are similar to those of Y Mus and V3795 Sgr. They were all discovered, except for A249, due to the interstellar extinction that reddened their $\mathrm{B}_{p}-\mathrm{R}_{p}$ colour above the 0.5 mag threshold used to create the initial star list (Sect. 2.1). We will widen up our search to bluer stars in future surveys.

The hydrogen-deficiency is real in all new dLHdC stars discovered. This is also confirmed by their near-IR spectra as reported by Karambelkar et al. (2022). However, surprisingly, many of new dLHdC stars have a degree of hydrogen-deficiency lower than most known $\mathrm{HdC}$ stars. We found traces of $\mathrm{H}_{\alpha}$ in 19 out of the 27: 3 belong to the Cold group ( $50 \%), 10$ belong to the Mild group $(\sim 77 \%)$ and 6 to the Warm one $(\sim 66 \%)$. They are listed in Table 3 with a scale from 0 to $2 "+$ " indicating the strength of the $\mathrm{H}_{\alpha}$ absorption line observed (see also Fig. 5 to 7 for a visualisation of them). One of the five previously known dLHdC stars, HD 148839, presented already such a characteristic. See Warner (1967) for a detailed discussion. Previously, abundance analysis of RCB and EHe stars have shown an apparent anti-correlation between the $\mathrm{H}$ and $\mathrm{Fe}$ abundances (Asplund et al. 2000, Fig.7). Here, our observations on $\mathrm{H}$ and $\mathrm{N}$ abun- 


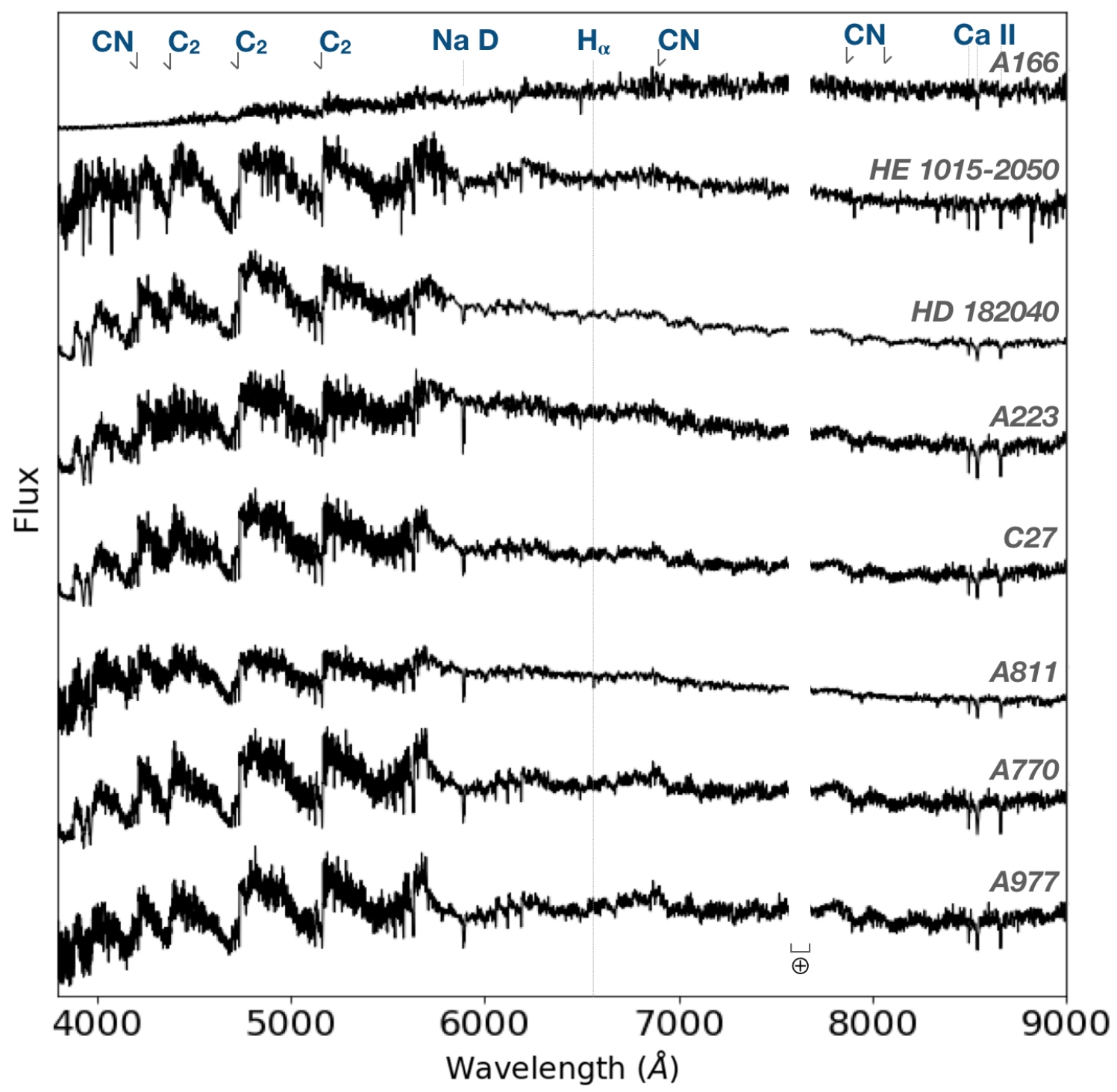

Fig. 5: Spectra, from 3800 to $9000 \AA$, of the dLHdC stars we considered as cold, $\mathrm{T}_{\text {eff }} \leq 5500 \mathrm{~K}$. The element related to the more prominent absorption lines and the expected position of $\mathrm{H}_{\alpha}$ are indicated with vertical dashed lines as well as various bandheads of $\mathrm{C}_{2}$ and $\mathrm{CN}$. The gaps in the spectra corresponding to telluric lines are indicated with the $\oplus$ symbol. The names of the corresponding stars are given on the right side. The ordinate is arbitrary.

dances for dLHdC stars go towards that trend. We even found a small emission $\mathrm{H}_{\alpha}$ line in the spectrum of the warm $\mathrm{HdC}$ star, F152, as well as some emission in the Ca II H\&K and triplet lines. RCB stars generally show weak or absent Balmer lines, but at least one of them, V854 Cen, shows significant hydrogen lines (Lawson \& Cottrell 1989; Kilkenny \& Marang 1989). Lawson (1992) has even detected $\mathrm{H}_{\alpha}$ in emission during a decline event. Recently, Tisserand et al. (2020) reported a similar spectroscopic behaviour with a new RCB star located in the Large Magellanic Cloud, named WISE_J054221.91-690259.3 (or MSX-LMC-1795). However, for F152, the $\mathrm{H}_{\alpha}$ emission is not correlated with a detected decline event as no such episode was seen on F152's ASAS-SN light curve at JD 2459452.3 (25th August 2021). At that time, F152 was even at a peak of its irregular photometric variability. Furthermore, we note that F75, another warm new HdC star, was also reported in the past to show some degree of emission lines as Stock \& Wroblewski
(1972) classified it as "Ge pec" for its spectral type which was therefore already seen as peculiar.

We also look for some traces of lithium in our mid-resolution spectra using the Li I 6707 absorption line. We found one strong occurrence in the spectrum of B563 and some hints for the possible presence of this line in the spectra of 11 others (see Table. 3) that would be of comparable strength than the one seen in HD 148839. They all need to be confirmed with higher resolution spectroscopy. Interestingly, the new HdC star B565 (for which we observed a small absorption line at the location expected for Li I 6707) has been observed by the GALAH survey (Buder et al. 2021) that uses the multi-fiber 2df/HERMES high-resolution (R 28 000) spectrograph (Object Id 170508006401160). They published a complete abundance analysis for that star and confirmed B565's high abundance of carbon and oxygen, as well as for lithium. Furthermore, their abundance analysis shows that it is a complete outlier in a diagram showing the abundance pattern 


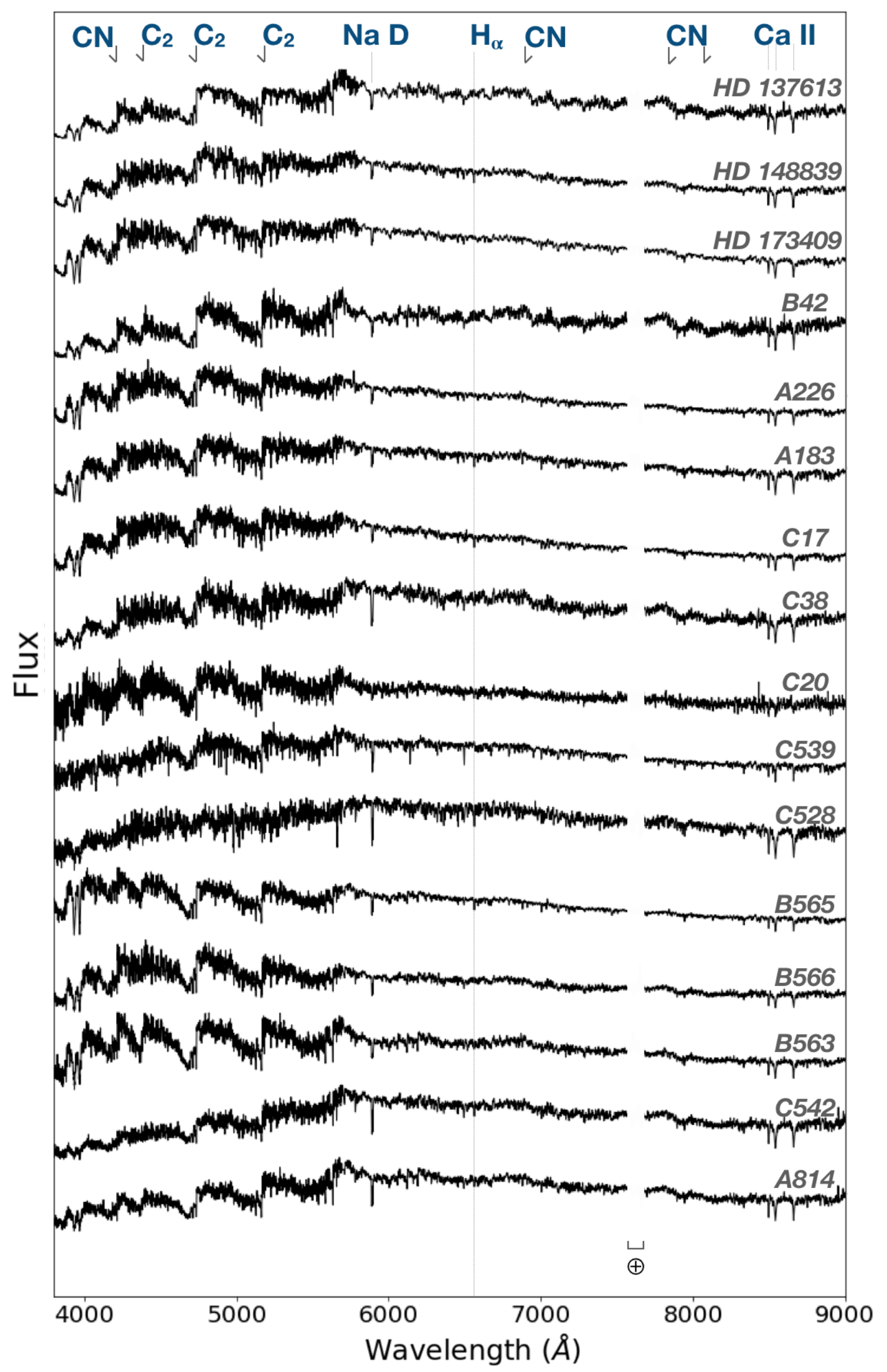

Fig. 6: Same as Fig 5 but for dLHdC stars we considered to have a mild temperature, $\mathrm{T}_{\text {eff }}$ between $\sim 6000$ and $\sim 7000 \mathrm{~K}$. 


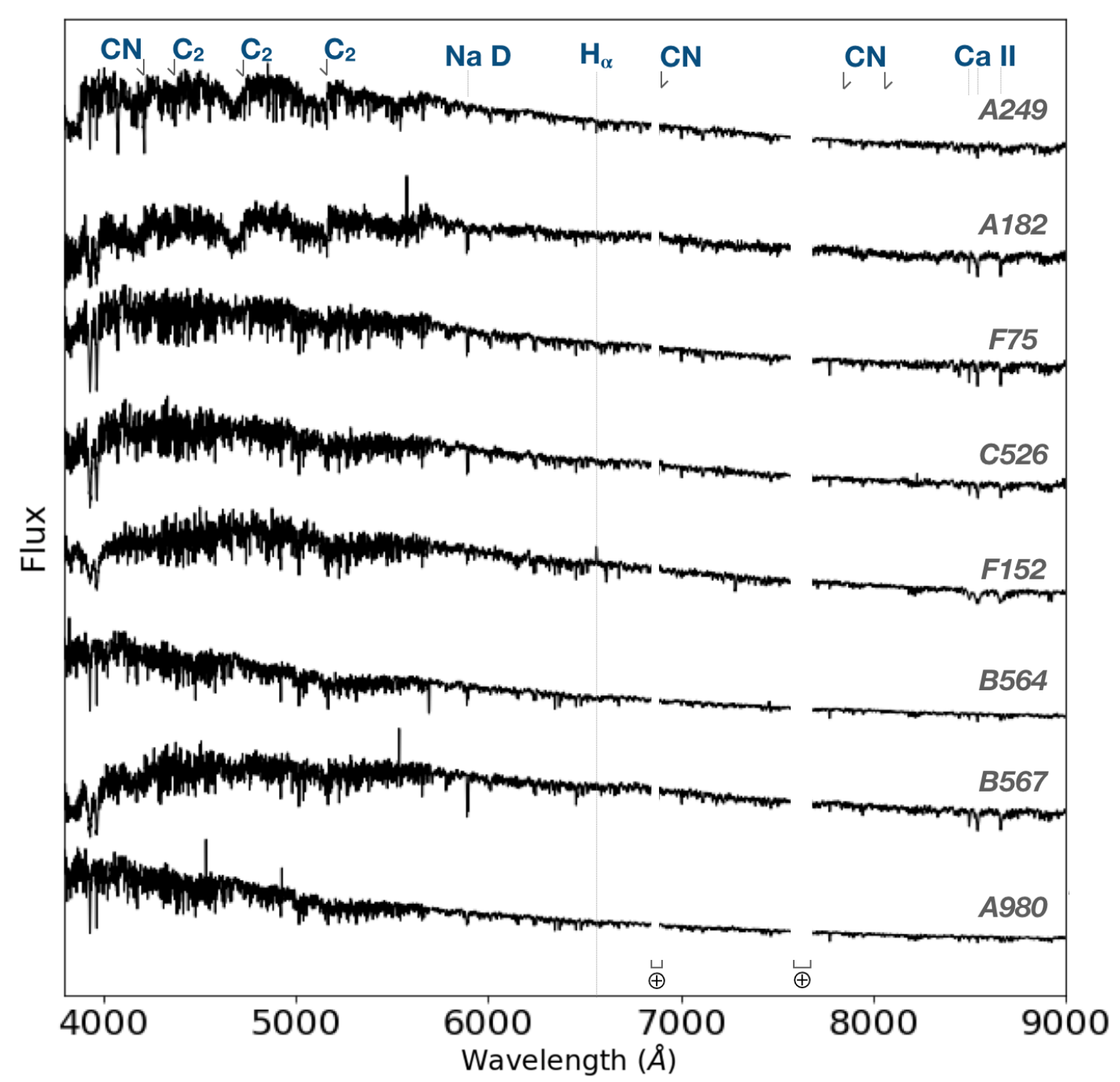

Fig. 7: Same as Fig 5 but for dLHdC stars we considered to have a warm temperature, $\mathrm{T}_{\text {eff }}$ between $\sim 7000$ and $\sim 8000 \mathrm{~K}$.

of iron vs. $\alpha$-process elements (with $[\alpha / \mathrm{Fe}] \sim 0.99$ and $[\mathrm{Fe} / \mathrm{H}] \sim$ 0.82 for B565) compared to the nearly 700000 stars they followed up.

Most RCB star atmospheres are known to have a low ${ }^{13} \mathrm{C} /{ }^{12} \mathrm{C}$ isotopic ratio, but this is not always the case (Rao \& Lambert 2008; Hema et al. 2012). We look for the presence of ${ }^{13} \mathrm{C}$ in the new dLHdC stars spectra, particularly in the blue region by comparing the ${ }^{13} \mathrm{C}^{12} \mathrm{C}$ absorption line located at $4744 \AA$ and the nearby $(1,0){ }^{12} \mathrm{C}^{12} \mathrm{C}$ at $4737 \AA$. It was difficult to carry on reliably such analysis in the red region as the $\mathrm{CN}$ band heads are weak. We found strong evidence for weak ${ }^{13} \mathrm{C}$ features in the spectra of A249, F75, C526, but also possibly in the spectra of A166.

Among all the newly discovered HdC stars, we found one new RCB star, C105. Its spectrum is presented in Figure 8 and compared to the one of ASAS-RCB-12, an RCB star of similar temperatures (i.e. $\mathrm{T}_{\text {eff }} \sim 6000 \mathrm{~K}$ ). They both show strong $\mathrm{C}_{2}$ features but some weak ones due to $\mathrm{CN}$. This is in contrast with the two other spectra presented, respectively from HD 175893 and V4017 Sgr, two RCB stars that are also of similar temperature. This indicates a lower nitrogen abundance in the atmosphere of C105 compared to more classical RCB stars. This was already seen in the atmosphere of all new dLHdC stars. C105 is a new RCB star because of the warm circumstellar dust we found to be surrounding it (see Fig. 15 and discussion in Sect. 4.4 and 4.5. However, we note that at the time of 2MASS' epoch (in 1998), C105's JHK magnitudes were not impacted by a warm thick dust shell as it was detected during the WISE survey in 2010 . Its colour indices, $(\mathrm{J}-\mathrm{H})_{0} \sim 0.14$ and $(\mathrm{H}-\mathrm{K})_{0} \sim 0.13 \mathrm{mag}$ are similar to the ones of dLHdC stars that show no IR excess (see Fig. 1). This explains also why C105 was not selected in the all-sky RCB star candidates list made by (Tisserand et al. 2020). C105 was in a phase of low dust production in 1998. We also found a light curve from the DASCH ${ }^{2}$ project covering most of the past century showing that $\mathrm{C} 105$ went under some characteristic decline phases at least four times, with two major ones observed in 1940 and 1972 (Fig. 4).

It is not surprising to find new RCB stars as 16 of the known ones (14 in groups A to D, and 2 in groups F and G) have passed through our selection cuts. These RCB stars did not show a high near-IR excess as no warm thick dust influenced the JHK photometry at the time of the 2MASS epochs.

2 DASCH: Digital Access to a Sky Century @ Harvard 


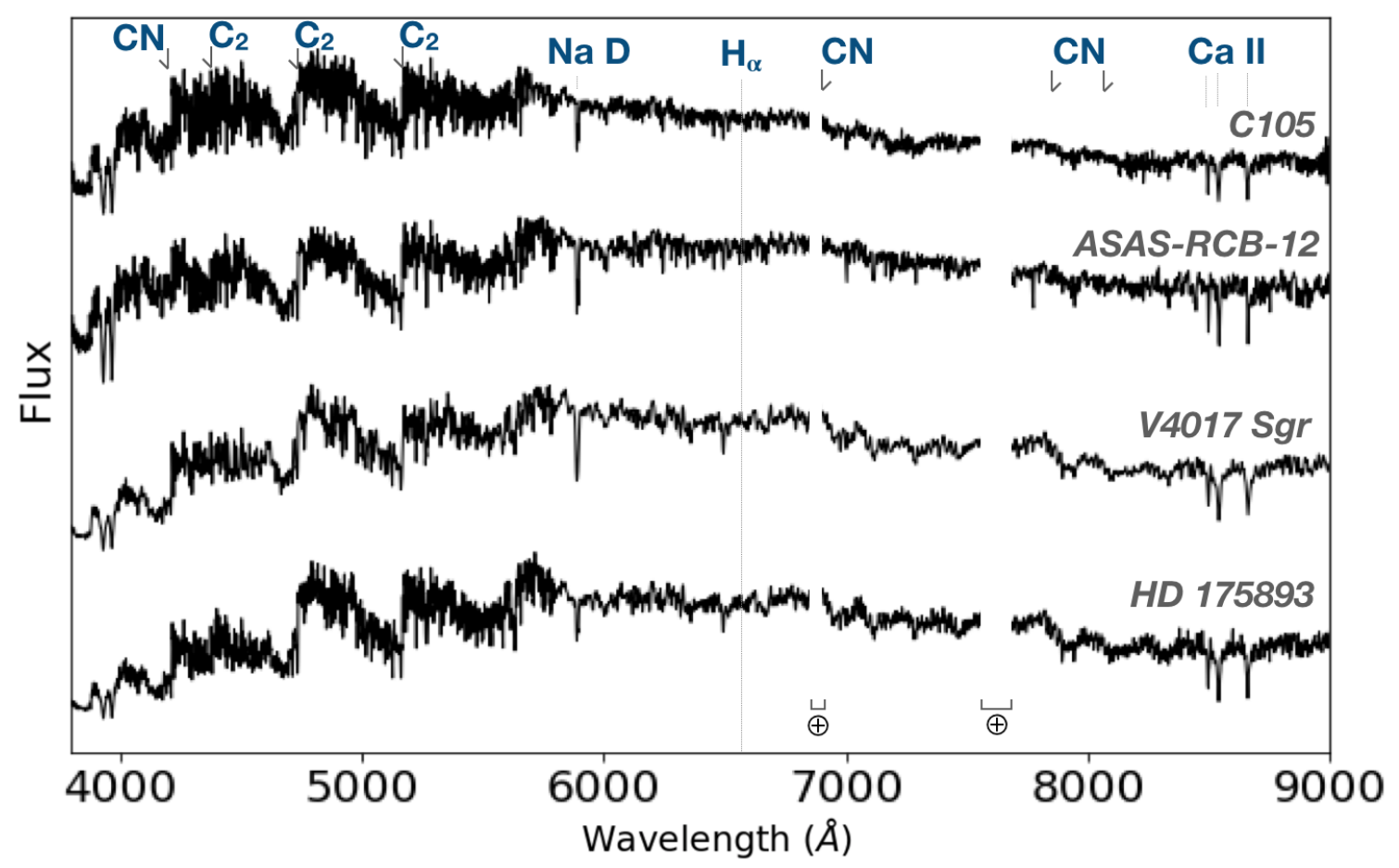

Fig. 8: Spectra, from 3800 to $9000 \AA$, of the newly discovered RCB star, C105, and HD 175893 that was considered for a long time as a dLHdC star but is in fact surrounded with a warm circumstellar dust shell as classical RCB stars. They are compared to known RCB stars whose spectra are very similar. The names of the corresponding stars are given on the right side. The ordinate is arbitrary.

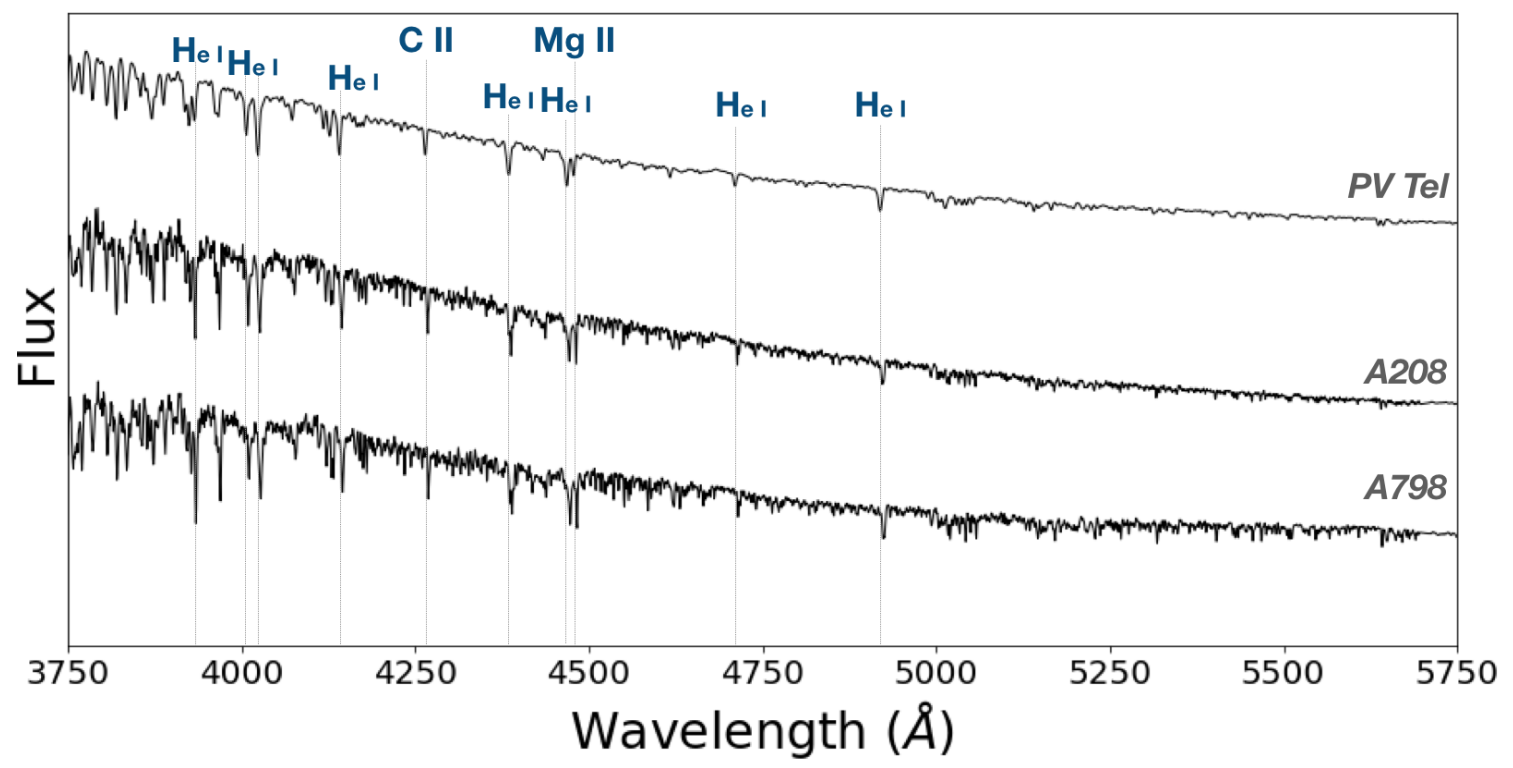

Fig. 9: Spectra, blue region from 3750 to $5750 \AA$, from 3800 to $9000 \AA$, of the newly discovered EHe stars and the known EHe star PV Tel that is of similar absolute brightness and temperature. The names of the corresponding stars are given on the right side. The ordinate is arbitrary.

\subsection{New extreme Helium stars}

Extreme Helium (EHe) stars are blue supergiant stars with surface temperatures ranging from 9000 to $35000 \mathrm{~K}$. They are thought to share an evolutionary connection with $\mathrm{RCB} / \mathrm{HdC}$ stars, being the subsequent phase of these last ones as they share similar spectroscopic characteristic due to an atmosphere poor in hydrogen but highly enriched in carbon (Jeffery 2008; Jeffery et al. 2011; Pandey et al. 2001).

Most known EHe stars (13 out of 21) were not part of the initial datasets of GAIA giant stars used in our analysis because of their uncorrected Bp-Rp colour index being bluer than 0.5 mag. Furthermore, of the remaining $8 \mathrm{EHe}$ stars, none has passed our criteria applied to the photometry recorded in the 2MASS and GAIA eDR3 catalogues. It is therefore surprising that we dis- 
covered two new EHe stars as they were not an objective of our search. Their discovery is due to a fortunate error. Indeed, a mistake in the process of calculating the $\mathrm{E}(\mathrm{B}-\mathrm{V})$ value was made for the stars belonging to group $\mathrm{A}$, which resulted in reddening values related to sky areas located between 20 to 40 arcmins away from each star. Therefore, some stars were observed spectroscopically while they should have been left out of the selection. The new EHe stars, A208 and A798, are two of them. From the distribution of these stars, the error is the equivalent of having observed randomly some stars located on the bluer side of the J-H vs H-K colour-colour diagram, within 0.05 mag from the selection limit. These stars are distributed homogeneously in the GAIA $\mathrm{M}_{G}$ vs (Bp-Rp) 0 colour magnitude diagram with a (Bp$\mathrm{Rp})_{0}$ colour ranging between -1.5 and $1.0 \mathrm{mag}$. Of the 656 stars originally selected in Group A with the wrong E(B-V) reddening estimate, 333 should not have been selected. Of this sample, 203 of them had no spectroscopic type defined in CDS/SIMBAD and were therefore confirmed targets for spectroscopic followup. We observed 51 of them before correcting the mistake, and by good fortune found 2 new EHe stars, whose coordinates and geometric distances are listed in Table. 2 .

The spectra, blue side, are presented in Fig. 9 with a direct comparison with the known EHe star, PV Tel. Most of the strong absorption lines that are detected are due to He I, but also C I and $\mathrm{Mg}$ II. Many more lines can be identified using the list published by Lynas-Gray et al. (1981) after studying PV Tel high resolution spectrum in detail. We calculated the visual absolute magnitudes and intrinsic colours $\left(\mathrm{M}_{V} \sim-3.60_{-0.21}^{+0.17}\right.$ and $\sim-4.16_{-0.27}^{+0.21} \mathrm{mag}$, (V$\mathrm{I})_{0} \sim-0.17$ and $\sim-0.04 \mathrm{mag}$, respectively for A208 and A798) and compared them to other EHe stars and HdC stars (see Fig. 10. They are located in a part of the HR diagram where we find other cool EHe stars, such as V2244 Oph, PV Tel, NO Ser, LSS 99, V1920 Cyg and V4732 Sgr.

Finally we check the light curves of both new EHe stars resulting from the ASAS-SN survey. We did not find any variability at the ASAS-SN's photometric resolution for A208, but report some variabilities with a peak to peak amplitude of $\sim 0.1$ mag and a periodicity of about 10 days for A798. This is confirmed by the monitoring by the CoRoT survey (COROT Team 2016). The high cadence light curve of A798, published among other stars observed in the faint star mode (monitoring between July and September 2011), shows well sampled irregular photometric variations, with a time scale between 5 to 10 days.

\section{Results and discussion}

\subsection{Galactic distribution of the new HdC stars}

The Galactic spatial distribution of all dLHdC stars is presented in Fig. 3 Five of them (C20, F75, F152, A166 and HE 10152050) are almost certainly located in the Galactic Halo, but most of the others are located around or inside the Galactic Bulge with geometric distances ranging between 6 and $12 \mathrm{kpc}$. We observed 216 candidates located around the Galactic centre ($45<1<45 \mathrm{deg}$ and $|\mathrm{b}|<15 \mathrm{deg}$ ) and found $21 \mathrm{dLHdC}$ stars, while similarly, we observed 135 candidates located along the Galactic disk $(180<1<300 \mathrm{deg}$ and $|\mathrm{b}|<15 \mathrm{deg})$ and found none. This is a clear indication that the dLHdC stars belong to the Bulge population, implying that it is an old population group of stars, as expected from their hydrogen-deficient nature. They share a similar sky distribution with RCB stars (see Tisserand et al. (2020, Fig.16).

We note that F152 is located on the line-of-sight of the Large Magellanic Cloud, half-way to the Milky Way's largest satellite, with a preferred median geometric distance of $\sim 24 \mathrm{kpc}$. It cannot be located within the LMC as its absolute brightness would be 1.5 mag brighter than the brightest HdC stars ever observed (see Sect. 4.2.

\subsection{Absolute brightness of the new HdC stars}

All newly discovered dLHdC stars range in apparent $\mathrm{G}$ brightness between the $\sim 10.5$ and $\sim 13.5 \mathrm{mag}$, with a peak at $\sim 12.5$ mag. About half of them are impacted by an E(B-V) interstellar reddening lower than $0.5 \mathrm{mag}$, while for the other half, the reddening extends up to $1.0 \mathrm{mag}$, being closer to the Galactic plane.

Most of the new dLHdC stars are located in the crowded area around the Galactic Bulge where the mean GAIA eDR3 parallax uncertainty for sources with five-parameter solutions increases notably due mainly to the small number of quasars identified at small Galactic latitudes (Lindegren et al. 2021a, Fig.2). The parallax SNR measured is lower than 10 for 25 out of the 27 new dLHdC stars. The exception being A183 and A811 detected with a parallax SNR respectively of $\sim 25.7$ and $\sim 10.5$ (Table 2). The parallax SNR go even down to values lower than 5 for 10 of them, which is nevertheless in the range of one can expect at the distance of Galactic Bulge if we consider the average GAIA eDR3 parallax uncertainties of $0.02-0.03$ mas for sources brighter than $\mathrm{G}<15$ mag.

We used for distance estimate the geometric distances inferred by Bailer-Jones et al. (2021) for the 1.47 billion GAIA eDR3 stars published with parallaxes. We listed these distances as well as their 1-sigma associated error in Table 2. Nineteen of the new dLHdC stars are located at a distance ranging between 6 and $11 \mathrm{kpc}$, supporting again their Galactic Bulge association. It is worth noting that two of the previously known dLHdC stars, HD 182041 and HD 137614, are the closest of all known HdC stars with distances of $\sim 884 \mathrm{pc}$ and $1.2 \mathrm{kpc}$ respectively, closer than the three closest RCB stars, R CrB, XX Cam and RY Sgr, whose respective distances are $\sim 1.3, \sim 1.3$ and $\sim 1.6 \mathrm{kpc}$.

We have obtained the absolute $\mathrm{V}$ magnitudes and the unreddened $(\mathrm{V}-\mathrm{I})_{0}$ colours for all dLHdC stars, the known EHe stars, some of the Magellanic RCB stars and 31 of the known Galactic RCB stars which have an eDR3 GAIA measured parallax with a SNR higher than 2 sigma (only 3 of them have a parallax SNR between 2 and 3). We kept only the RCB stars for which we were able to estimate convincingly their maximum brightness in both $\mathrm{V}$ and I photometric bands. We used the light curves of the OGLE surveys for the Magellanic RCBs (Udalski 2008) and mainly the ASAS-3 and ASAS-SN surveys for the Galactic RCB stars, in association with the AAVSO database for the bright ones. For the dLHdC and EHe stars, we used the ATLAS AllSky Stellar Reference Catalog (Tonry et al.2018) to recalculate a V and I magnitudes, and used the ASAS-3 and ASAS-SN light curves to confirm the $\mathrm{V}$ magnitudes for all dLHdC stars. For the four really bright known dLHdC stars, we used mostly the magnitudes provided either by the UBVRIJKLMNH Photoelectric Catalogue (Morel \& Magnenat 1978) or the catalogue of Stellar Photometry in Johnson's 11-color system (Ducati 2002). To correct for the interstellar reddening, we primarily used the $3 \mathrm{D}$ dust map produced by Green et al. (2019), but then used the E(BV) reddening values produced by Schlafly \& Finkbeiner (2011) over the entire sky if information was not available in Green et al.

The result is presented in the $\mathrm{M}_{V}$ vs $(\mathrm{V}-\mathrm{I})_{0}$ colour magnitude shown in Figure 10 One can see that the EHe stars, which are thought to be the final phase of $\mathrm{HdC}$ stars, are indeed bluer than 

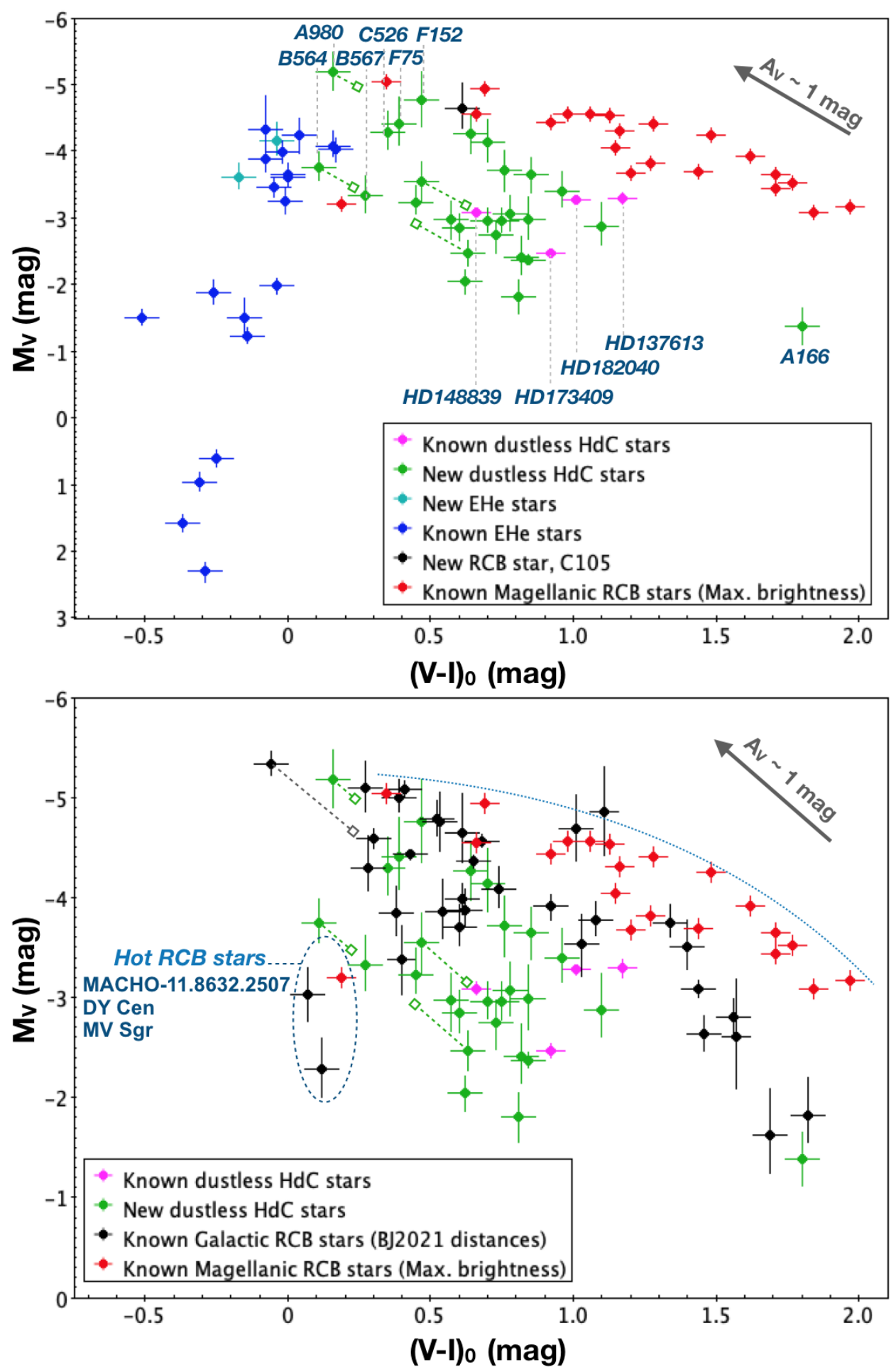

Fig. 10: Colour-magnitude diagrams $\mathrm{M}_{V}$ vs $(\mathrm{V}-\mathrm{I})_{0}$ with all dLHdC stars represented as well as most known EHe stars, some Magellanic RCB stars and the Galactic RCB stars for which a GAIA eDR3 parallax was reported with SNR $>2$ and for which we were able to measure convincingly their maximum brightness in both $\mathrm{V}$ and I photometric bands. For all Galactic objects, we used the median geometric distances inferred by Bailer-Jones et al. (2021) and the error bars on the luminosity scale reflect their associated $68 \%$ confidence interval. Top: all stars except the known Galactic RCB stars are represented. Bottom: zoom on the brightest part of the diagram with the EHe stars removed and the Galactic RCB stars added. An interstellar reddening arrow representing an extinction of $1 \mathrm{mag}$ in $\mathrm{V}$ is represented in both figures. The dashed lines, parallel to the extinction arrows, that are each associated to a Warm RCB star (Y Mus), two Warm dLHdC stars (A980 and B564) and two Mild dLHdC stars (C17 and A182) correspond to the reddening over- and under- correction we consider to have applied after estimating the stars respective temperature from their spectrum. The location of the hot RCB stars, MACHO-11.8632.2507, DY Cen and MV Sgr are indicated with an ellipse. The dotted curved is used to illustrate the maximum absolute $\mathrm{M}_{V}$ magnitudes recorded for each bin of $(\mathrm{V}-\mathrm{I})_{0}$ colour index. 
any HdC stars and are getting even bluer while decreasing in magnitudes. Surprisingly, we found that the known dLHdC stars and the majority of the new $\mathrm{dLHdC}$ stars are intrinsically fainter than the Magellanic RCB stars but also most of the Galactic RCB stars by about $1.5 \mathrm{mag}$. Originally, Alcock et al. (2001) and Tisserand et al. (2009) brought to light a relationship between the Magellanic RCB stars maximum brightness and their respective visual colour, but no such large spread were then observed in brightness. The outliers which are fainter RCB stars that did not follow the relationship in Tisserand et al. (2009, Fig.3) were then explained by a need of a supplementary carbon extinction coming from the circumstellar shell and consequently by not having observed the maximum magnitudes. Here, we have accumulated enough measurements in the light curve of each RCB stars used in the diagrams to be convinced of our measures of their respective maximum magnitudes. Overall, we found that the absolute brightness of HdC stars span over $\sim 3$ magnitudes for a colour $(\mathrm{V}-\mathrm{I})_{0} \sim 0.5 \mathrm{mag}$.

Most of the dLHdC stars have a colour $(\mathrm{V}-\mathrm{I})_{0}$ index lower than $1.0 \mathrm{mag}$, indicating temperatures between 5000 and 8000 $\mathrm{K}$. We looked for reasons why we would have potentially missed their cooler counterparts, but we did not find strong arguments for a selection effect. First, these colder objects are expected to be intrinsically fainter than their warmer ones, but from the second cut applied (Fig. 2, right), the range in colour and brightness selected should be wide enough to be able to pick up some of this missing population of cold dLHdC stars even if they were $1 \mathrm{mag}$ fainter than their warmer counterparts. This is supported by our discovery of A166, the coldest new HdC of our sample (see the discussion on that star, at Sect.4.6). Second, we looked at the effect of our first pragmatic selection cuts applied in the J-H vs H-K diagram (Eq. 1), and we realised afterwards that the distribution of the newly discovered dLHdC stars in that diagram depends on their effective temperature as one can see in Figure 11. There is no clear evidence that cool dLHdC stars candidates could potentially have been removed at that stage.

We found that many dLHdC stars have an absolute magnitude around $\mathrm{M}_{V} \sim-3 \mathrm{mag}$ and are distributed in a region of the HR diagram where there are no known RCB stars. Furthermore, the six brightest dLHdC stars $\left(\mathrm{M}_{V}<-4 \mathrm{mag}\right)$, i.e., C526, F152, F75, A980, A814 and C542, are not distributed in a sky area towards the Galactic Bulge like most of the other fainter dLHdC stars (see Fig. 12). Those dLHdC stars could thus be an underlying population of real "long-term" dustless HdC stars.

On the warmer side, we found that three Hot RCB stars, MACHO-11.8632.2507, DY Cen and MV Sgr, are located in the same part of the diagram with similar absolute magnitudes $\mathrm{M}_{V} \sim-3$ mag and colours $(\mathrm{V}-\mathrm{I})_{0} \sim 0.15 \mathrm{mag}$, as expected from their effective temperatures that are higher than $12000 \mathrm{~K}$. Four warm HdC stars were found to have colour indices lower or similar to these Hot RCB stars, they are the two RCB stars, Y Mus and ASAS-RCB-10, and the two new dLHdC stars, B564 and A980. We have almost certainly applied an interstellar reddening correction that is too high for these objects whose effective temperature is about $8000 \mathrm{~K}$. We indicated in the colour-magnitude diagram the over-correction needed to reach a more reasonable colour of $(\mathrm{V}-\mathrm{I})_{0} \sim 0.25 \mathrm{mag}$.

Similarly, on top of the 1- $\sigma$ error bars that represent our best knowledge of the uncertainties related to distance, apparent maximum brightness and colours, one needs to imagine the effects of an over- and/or under- correction of interstellar dust reddening in the $\mathrm{M}_{V}$ vs $(\mathrm{V}-\mathrm{I})_{0}$ colour magnitude. Extinction vectors are indicated in both diagram of Figure 10 . We do not expect a drastic change in the overall distribution of HdC stars. However, we

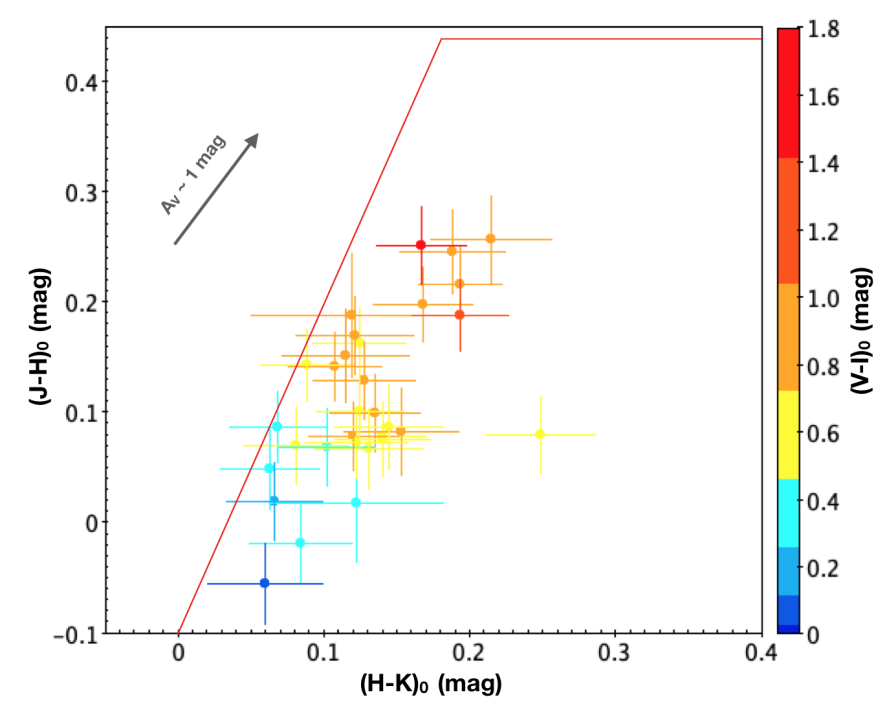

Fig. 11: Colour-colour J-H vs H-K diagram with 2MASS magnitudes corrected for extinction showing the positions of all dLHdC stars, similar to Figure 1. Here the dots and error bars are colour coded with the respective $(\mathrm{V}-\mathrm{I})_{0}$ colour found for each star. The selection cuts applied are represented with red solid lines.

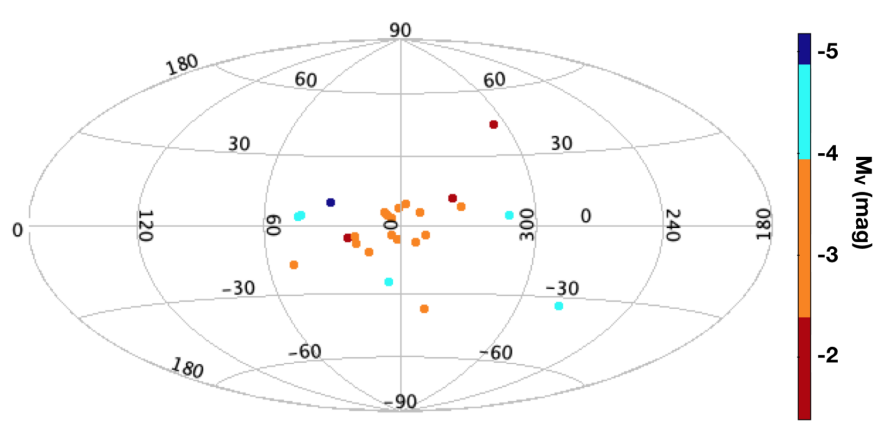

Fig. 12: Spatial distribution in Galactic coordinates of the $27 \mathrm{dL}-$ HdC stars discovered, colour coded with their respective absolute magnitude $\mathrm{M}_{V}$. A166 and the bright dLHdC stars are not located towards the Galactic Bulge sky area, where we found most of the population of fainter dLHdC stars $\left(\mathrm{M}_{V} \sim-3 \mathrm{mag}\right)$.

noted that for few of them one should expect a shift up to 0.2 mag in colour, when one compares their $(\mathrm{V}-\mathrm{I})_{0}$ colour indices with their temperature estimates based on their respective spectra. We found, for example, that $\mathrm{C} 17$ (classified as a Mild dLHdC star) has an estimated (V-I) $)_{0}$ colour index of 0.47 mag that is too blue for its temperature. Inversely, A182 (classified as a Warm dLHdC star) has an estimated (V-I) $)_{0}$ colour index of 0.63 mag that is too red and a higher reddening correction should thus be applied. Finally, we think that the warm dLHdC star, A182, should be bluer than its derived colour of $(\mathrm{V}-\mathrm{I})_{0} \sim 0.61 \mathrm{mag}$.

F152 and F75 are two new HdC stars for which the GAIA eDR3 parallax SNR is lower than 3, but their geometric distance estimate associated with a larger 1-sigma error is still statistically valid. We plotted those two in the above colour-magnitude diagram (Fig. 10) to illustrate their positions compare to the other dLHdC stars as we have evidence of a dust production activity for these two stars despite the observational lack of warm circumstellar dust shell around them (see Sect 4.5). They both 
present a spectrum which indicates a warm effective temperature of $\sim 7500 \mathrm{~K}$ and their position in the HR diagram correspond to that of RCB stars of the same temperature with absolute $\mathrm{M}_{V}$ magnitudes ranging between -4.4 and -5 mag for a $(\mathrm{V}-\mathrm{I})_{0}$ colour index of $\sim 0.4$ mag.

Also, in the curiosity cabinet, we note that A980 and B564 are the two warmest dLHdC stars we found, with a temperature around $\sim 8000 \mathrm{~K}$. They present very similar spectra (Fig. 7), but their absolute $\mathrm{M}_{V}$ magnitudes depart by $1.5 \mathrm{mag}$, A980 being the brightest. We couldn't distinct any differences in the absorption lines equivalent widths within our spectroscopic resolution.

\subsection{Interpretation in the framework of the double degenerate scenario}

In the double degenerate scenario, Saio \& Jeffery (2002, Fig.2) have presented the evolution of an $\mathrm{HdC}$ star in the HR diagram following the merger of a CO-WD with an He-WD for different initial total masses and WD mass ratio. They showed that such an object becomes brighter and warmer after an initial cool phase following the merger and that the maximum luminosity should increase with the initial total mass. They calculated a difference in maximum luminosity of $\sim 2.1$ mag between a WD system with a total mass of $0.6 \mathrm{M}_{\odot}$ and one of $0.9 \mathrm{M}_{\odot}$. In light of such models, here, we are possibly seeing, for the first time, the evolutionary sequences of WD-binary mergers with a large range of initial total mass.

The mass distribution is predicted by population synthesis simulations of close binary systems in StarTrack (Belczynski et al. 2008, Ruiter et al. 2014) after various mass transfer phases. The double white dwarfs merger types with a high total mass (CO-CO, $\mathrm{CO}-\mathrm{ONe}$ or $\mathrm{ONe}-\mathrm{CO}$ ) and the ones with a low total mass $(\mathrm{He}-\mathrm{He})$ are neglected as a reasonable channel to be $\mathrm{HdC}$ progenitor stars. Only the systems with intermediate total WD masses are used. In such, double WD binaries in which a 'hybrid' COHe WD (Tutukov \& Yungelson 1996, Zenati et al. 2019) forms before a CO WD contributes to the massive end of the total mass distribution, with an average merger mass of $\sim 0.9$ $\mathrm{M}_{\odot}$ (Karakas et al.2015, Fig.1). These types of WD mergers are also more numerous compared to the other WD merger types we consider and are formed through a stable Roche lobe overflow (RLOF) phase when the primary star fills its Roche lobe on the MS or in the Hertzsprung gap, continuing through to when the star is a red giant, followed later by a common envelope phase where the secondary star loses its envelope as a red giant or an AGB star. Some systems undergo a further mass transfer phase, typically dynamically stable, where the donor (having lost its hydrogen envelope previously in RLOF) is on the helium main sequence. The merger mass distribution from StarTrack is presented in Fig. 13. The distribution covers a total mass range between $\sim 0.6$ and $\sim 1.05 \mathrm{M}_{\odot}$ and has a bimodal structure. The notation $\mathrm{HybCO}$ indicates that the initially more massive star on the ZAMS formed the hybrid WD, where COHyb indicates the initially more massive star on the ZAMS formed the CO WD. For clarity, we show the less-populated WD merger channels grouped together (in orange). Mergers between a helium WD and a hybrid WD produce average merger masses on the lower end, $\sim 0.65 \mathrm{M}_{\odot}$, thus may contribute more to the less luminous population of HdC stars. Here, a low metallicity that is more along the lines of what is found in the SMC was used, though the main distribution features persist at solar metallicity.

Though likely not all of the WD merger channels we explored contribute to the HdC star population (e.g. some might contribute to low-luminosity thermonuclear supernovae as ex-

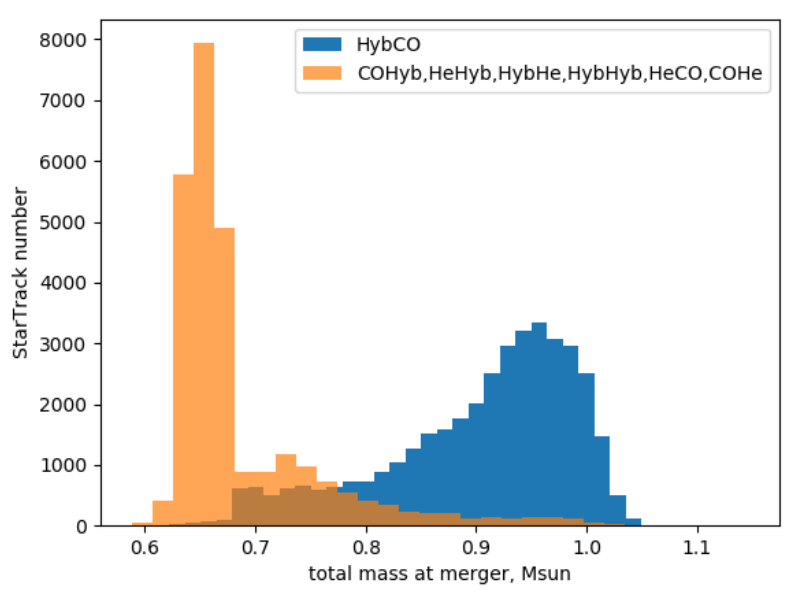

Fig. 13: Plausible mass distribution of the total WD systems that are considered to be the progenitor of HdC stars. The contribution from different WD merger types are considered in all combination between a hybrid, a $\mathrm{CO}$ and a $\mathrm{He}$ WD (except $\mathrm{CO}+\mathrm{CO}$ and $\mathrm{He}+\mathrm{He}$ ). For illustration purpose, we separated the more massive channel made of a hybrid and a CO WD merger (blue) to all other ones (orange) listed in the legend. The overall distribution is bi-modal.

plained in Crocker et al. (2017) or Pakmor et al. (2021)), the different formation scenarios and resulting WD merger masses makes it rather tempting to assign different formation pathways to the different $\mathrm{HdC}$ star populations.

We find also that WD mergers from the more massive HybCO channel have as well an interesting bimodal delay time distribution with about $40 \%$ of systems merging within $2 \mathrm{Gyr}$ after star formation (with the first mergers occurring $250 \mathrm{Myr}$ after starburst), while around $60 \%$ take at least 5 Gyr after star formation to merge with some systems capable of merging beyond a Hubble time. Those with shorter delay times encounter a common envelope phase when the mass-losing star is a red giant, whereas those with longer ( $>5 \mathrm{Gyr}$ ) delay times encounter the common envelope phase when the mass-losing star is on the AGB. In the hypothesis that all WD mergers from this channel form $\mathrm{HdC}$ stars, we thus would expect to find those to be present both in the Galactic bulge as well as regions of active star formation. We do not have strong evidence of a large population of $\mathrm{HdC}$ stars located in the spiral arms, though their progenitors may still be actively forming there. Using the 3D dust map of Green et al. (2019), we noted that the 3 following RCB stars, GU Sgr, UV Cas and V2552 Oph, and the dLHdC star A223 have a galactic position where we found a large increase of dust reddening consistent with a location within or near a spiral arm.

Interestingly also, as expected in the double degenerate scenario, we can interpret that the upper luminosity envelope we are seeing in the lower panel of Figure 10 formed by the maximum absolute magnitudes recorded for each bin of $(\mathrm{V}-\mathrm{I})_{0}$ colours, starting at $\left(\mathrm{M}_{V},(\mathrm{~V}-\mathrm{I})_{0}\right) \sim(-5.2 ; 0.3) \mathrm{mag}$, and ending at $\sim(-$ 3.3 ; 2.0) mag, as the evolution sequence of $\mathrm{HdC}$ stars with the highest total initial mass possible to form such supergiant stars. This envelope is illustrated as a dotted curve in Figure 10 and corresponds to this simple second order polynomial function $\mathrm{M}_{V}=\mathrm{A}+\mathrm{B} \times(\mathrm{V}-\mathrm{I})_{0}+\mathrm{C} \times(\mathrm{V}-\mathrm{I})_{0}^{2}$ with $\mathrm{A}=-5.08, \mathrm{~B}=-0.539$ and $\mathrm{C}=0.711$. Above that mass threshold, the WD mergers may result in supernovae (Sim et al. 2010; Ruiter et al. 2011, 2014). Cu- 
riously though, for cool temperatures (i.e. $(\mathrm{V}-\mathrm{I})_{0}>1.0 \mathrm{mag}$ ), this envelope is made only by Magellanic RCB stars, all cold Galactic RCB stars being fainter. We cannot explain such differences by any issues due to the calibration of the photometric $\mathrm{V}$ and I bands, the interstellar reddening correction (most cool Galactic RCB stars being located towards low reddening sky area) or the distance estimates. It is based on 7 cool Galactic RCB stars for which we succeeded to gather enough information. More work will be needed on the $60+$ other cool Galactic RCB stars known to confirm this observation. One could thus interpret this contrast in term of difference of stellar population between both Galaxies, resulting in two similar total initial mass regimes but two distinct WD mass ratios. Such a scenario is presented by Saio \& Jeffery (2002, Fig.2) in the specific case of a total initial mass of $0.9 \mathrm{M}_{\odot}$. The evolution track resulting from of a $0.6 \mathrm{M}_{\odot} \mathrm{CO}-\mathrm{WD}$ that accreted a $0.3 \mathrm{M}_{\odot} \mathrm{He}-\mathrm{WD}$ is brighter at cool temperatures than the one resulting from a $0.5 \mathrm{M}_{\odot}$ mass CO-WD that accreted a $0.4 \mathrm{M}_{\odot}$, but reach similar luminosities at warmer temperatures before reaching the EHe phase. Furthermore, a theoretical study on the metallicity dependence of the initial-to-final mass relation made by Romero et al. (2015) shows that metal-rich progenitors result in less massive WD remnants, due to an enhancement of the mass-loss rates associated with high metallicity values. One can expect differences up to $\sim 0.1 \mathrm{M}_{\odot}$ in the final WD mass. This effect can thus well explained our observations with Magellanic RCB stars being more massive than their Galactic counterparts due to the difference of metallicity between both populations (Magellanic stellar population have a lower metallicity).

\subsection{Photometric variabilities}

We studied the light curves of all dLHdC stars aggregated from the following four monitoring surveys: ASAS-3 (Pojmanski 1997), ASAS-SN (Shappee et al. 2014; Kochanek et al. 2017), Catalina (Drake et al. 2012) and Palomar Gattini IR 3 (De et al. 2020; Moore \& Kasliwal 2019). The ASAS-SN light curves were used to study variabilities on a small time scale, while the others were inspected to check for the presence of possible declines.

The striking first result from this study is that most of the dLHdC stars (19 out of the 32) show no variability at the photometric resolution of the ASAS-SN survey, while, for the other 13 , we detect only some low peak-to-peak amplitude variabilities (between 0.05 to $0.15 \mathrm{mag}$ ) on time scales of the order of 10 days (see Table 4). This is different to the irregular oscillations observed in RCB stars, which vary, when they are at maximum luminosity, between 0.2 and 0.4 mag on a time scale between 20 to 60 days. This indicates clear physical differences between these two populations of $\mathrm{HdC}$ stars.

The low amplitude photometric variability of the dLHdC stars is supported by other surveys. We note that using the Hipparcos dataset, Rimoldini et al. (2012) found that HD 137613 presents variabilities with a peak-to-peak amplitude of 0.05 mag on a time scale of 0.1 day, however, this is not confirmed by the KELT survey (Oelkers et al. 2018) that did not find variabilities greater than $0.04 \mathrm{mag}$ for that star on time scales ranging from 30 mins to 1 day. As to the brightest dLHdC star, HD 182040, $\mathrm{Ri}-$ moldini et al. (2012) classified it as a variable star with a peak-topeak amplitude of 0.04 mag and a period of $\sim 1.3$ days. This variability is confirmed by the Mascara monitoring survey of bright stars (Burggraaff et al. 2018) that reports a clear variability sig-

\footnotetext{
3 The Gattini IR light curves are available at this URL: http://rcb.iap.fr/tracking_dLHdC/Gattini-IR/
}

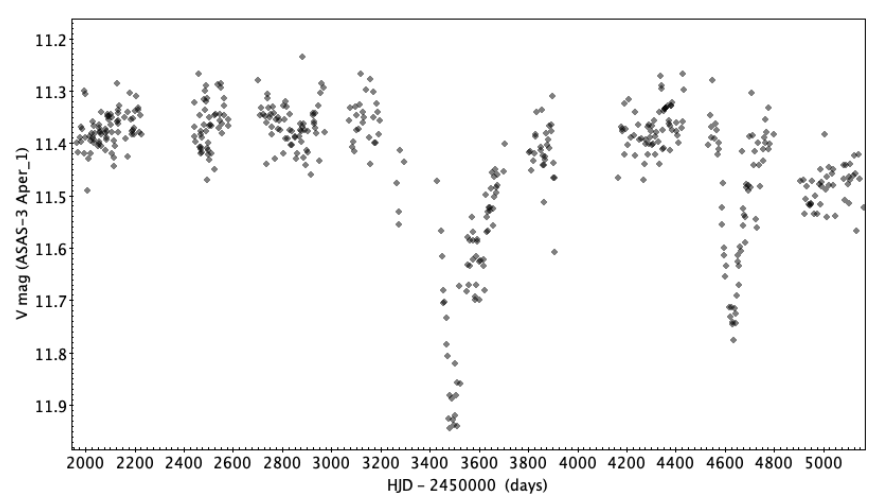

Fig. 14: ASAS-3 V band light curve of F75. Two small photometric declines were observed.

Table 4: Photometric variabilities observed in the ASAS-SN light curves of dLHdC stars and RCB stars.

\begin{tabular}{ccc}
\hline Stars & $\begin{array}{c}\text { Time scale } \\
\text { (days) }\end{array}$ & $\begin{array}{c}\text { Peak-to-peak } \\
\text { amplitude (mag) }\end{array}$ \\
\hline \multicolumn{3}{c}{$d$ LHdC stars } \\
\hline A166 & $\sim 25 \& \sim 200$ & 0.15 \\
A182 & $\sim 15$ & 0.15 \\
A223 & $\sim 10$ & 0.1 \\
A249 & $10-20$ & 0.05 \\
A770 & $\sim 10$ & 0.1 \\
A980 & $10-20$ & 0.1 \\
B42 & $10-40$ & 0.1 \\
B564 & $\sim 10$ & 0.08 \\
B566 & $\sim 10$ & 0.12 \\
C27 & $10-40$ & 0.15 \\
C526 & $10-40$ & 0.15 \\
F75 & $10-25$ & 0.15 \\
F152 & $30-60$ & $0.1-0.2$ \\
\hline \multicolumn{3}{c}{ RCB stars } \\
\hline C105 & 40 \\
HD 175893 & 20 & 0.4 \\
\hline
\end{tabular}

nature, that is irregular, but with some oscillations observed with 0.04 mag peak-to-peak amplitude and $\sim 9$ days duration. Concerning the new dLHdC stars, (Oelkers et al. 2018) list A183, C20, A980 and A166 as non variable stars on a short time scale of less than one day with amplitudes that would be respectively higher than $\sim 0.01, \sim 0.03, \sim 0.025$ and $\sim 0.05$ mag. However, on a longer time scale, the ATLAS survey (Heinze et al. 2019) reports that A166 could be an irregular variable with $\sim 0.1$ mag variation, consistent with our observations from its ASAS-SN light curve (Tab. 4). They also report that A223, B566, C542, A977 show long time scale variations of between 0.07 and 0.1 mag amplitude, without being successful in a clear classification of variability class. Finally, the TASS Mark IV photometric survey of the northern sky (Droege et al. 2006) list A980 with a variability index indicating significant change in brightness, and B42 is listed in the ASAS-SN catalog of variable stars (Jayasinghe et al. 2018) to present variabilities of $\sim 0.09$ mag amplitude on time scale of $\sim 41$ days. We also confirm these observations. For B42, we are observing also multiple irregular variations on time scale of $\sim 10$ days (Tab. 4).

The ASAS-SN light curve of the new RCB star, C105, shows variabilities typical of RCB stars with a peak-to-peak amplitude of $\sim 0.4$ mag and time scale of 40 days. It was listed in the ASAS- 
SN catalogue of variable stars as a non periodic variable star of $\sim 0.26$ mag amplitude (Jayasinghe et al. 2018). This is also the only object discovered in the present analysis that was listed as a variable star in the SIMBAD database, due to the high frequency light curve published by the HATNET variability survey (Hartman et al. 2004) that shows a smooth variation of $0.12 \mathrm{mag}$ amplitude over 30 days. Finally, Heinze et al. (2019) reported variations of $\sim 0.35 \mathrm{mag}$ detected by the ATLAS survey. We also looked at the ASAS-SN light curve of HD 175893 and found variabilities of $0.3 \mathrm{mag}$ on time scale of 20 days (Tab. 4).

We did not detect photometric declines in any of the dLHdC stars except one. Indeed, the ASAS-3 light curve of F75 present two declines at JD 2453450 and $\sim 2454600$ days that last respectively $\sim 300$ and $\sim 150$ days, with a maximum obscuration of $\sim 0.55 \mathrm{mag}$ in the first event and $\sim 0.40 \mathrm{mag}$ in the second (See Fig. (14). The final part of the first decline and the second one entirely are even confirmed by the Catalina survey. These declines indicate certainly an ongoing phase of dust production. This will be discussed below while studying F75 mid-IR WISE photometry. F75 could be in the process of starting its phase as an RCB star.

\subsection{Dust around the new dLHdC stars?}

RCB stars are the dusty version of HdC stars. They are known to be actively producing dust made of amorphous carbon dust grains and thus are surrounded by warm circumstellar shells (Feast et al. 1997, García-Hernández et al. 2011, Tisserand 2012). We are seeing RCB stars producing dust at all temperatures, from the very cold $\left(\mathrm{T}_{e f f} \sim 4000 \mathrm{~K}\right)$ to the very hot ones $\left(\mathrm{T}_{\text {eff }}>12000 \mathrm{~K}\right)$. The mechanisms needed to make this dust seem therefore not to be much influenced by temperature. Also, we are seeing few RCB stars surrounded by multiple shells. We observed such features in the spectral energy distribution (SED), as seen by WISE in 2010, of the three following hot RCB stars, MV Sgr, DY cen and MACHO-11.8632.2507, but also of a cold and of a warm RCB star, that are WX CrA and UW Cen respectively (Tisserand 2012). It shows that RCB stars, of any temperature, could stop or slow down their production of dust for a period of time, before restarting it again. Finally, Montiel et al. (2018) presented high-resolution mid-IR images of few RCB stars showing the existence of extended cold shells. The dust production could be made throughout the lifetime of an RCB star.

The four historic dLHdC stars are known to be deprived of any warm circumstellar inner dust shell, unlike RCB stars, since the data release of the WISE mid-IR photometry and thus since the study of their SED up to 22 microns (Tisserand 2012). Before that, we simply knew that these four HdC stars were never detected to undergo large photometric declines. Consequently, one can imagine they have either not started a dust production process yet or perhaps the last one stopped long ago. At longer wavelength, searches were made to detect the possible remains of cool extended dust shells. This is made difficult by the background dust emission that is often dominant at low Galactic latitude. First, using the IRAS satellite catalogue, Walker (1985) searched for IRAS flux but HD 182040 was only detected at 12 $\mu \mathrm{m}$. Then, recently, (Montiel et al.2018) studied HD 173409 using the Herschel/PACS and SPIRE instruments, and found that no nebulosity is visible in the images taken between 70 and 500 microns. Cold dust shells were however discovered in most RCB stars they observed, on top of the existing warm inner shell. This is another clear difference between the two population of $\mathrm{HdC}$ stars.

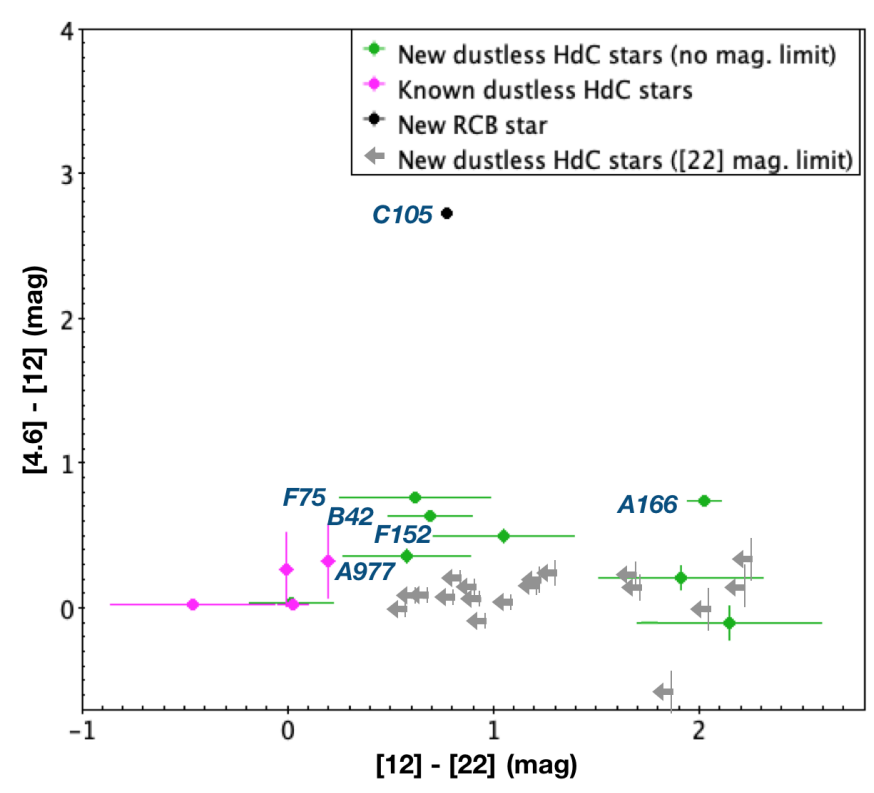

Fig. 15: Colour-colour [4.6] - [12] versus [12] - [22] diagram using WISE ALLWISE mid-IR magnitudes, see Tisserand et al. (2020, Fig.1) for a direct comparison with Known RCB stars. The new RCB star, C105, is indicated with a black dot. The new $\mathrm{dLHdC}$ stars are indicated with green dots and grey arrows for the ones published with a magnitude limit in the [22] passband. The known dLHdC stars are shown with purple dots.

We looked at the mid-IR WISE colours of each new HdC star discovered to detect the possible presence of circumstellar shells. We found that, contrary to all known Galactic RCB stars located in similar crowded areas, most of the new HdC stars have no detection reported in the reddest of the four WISE bands: 18 out the $27 \mathrm{dLHdC}$ stars present indeed a magnitude limit in the [22] photometric band. Additionally, no entry in the WISE AllSky and ALLWISE catalogues was found for B566. The nearest WISE source is located at 2.2 arcsec and corresponds to the blend of B566 with a nearby object. Finally, the faintest known dLHdC star, HE 1015-2050, was reported with two magnitude limits in the two redder WISE bands. Both objects will not be part of our study. On top of the four brightest known dLHdC stars, 8 of the new discovered ones present a valid measurement in all four WISE passbands.

Our study can be summarised with the WISE colour-colour [4.6]-[12] vs [12]-[22] presented in Figure 15. First, one can immediately see that C105 stands out as it is located in a part of the diagram away from the main locus of dLHdC stars. This is a new RCB star. Its position corresponds to the area expected for a typical RCB star that has formed a thick circumstellar dust shell of temperature $\sim 500 \mathrm{~K}$ (see Tisserand et al. (2020, Fig.1) for a direct comparison with Known RCB stars). Second, all but 5 dLHdC stars present a mid-IR colours consistent with no excess. These five HdC stars are A977, F75, F152, B42 and A166. They present a [4.2]-[12] colour higher than 0.3 mag, F75 having the reddest colour with $\sim 0.76 \mathrm{mag}$. These excesses, if confirmed, should indicate the presence of thin dust shells.

We mentioned in the preceding section that the warm $\mathrm{HdC}$ star, F75, has undergone two small photometric declines indicating a current phase of dust production. This is supported by its WISE colours. This suggests that F75 is certainly an HdC star that is in a transition time, as it is starting its RCB phase. Interestingly, this is the first known HdC star of the sort. As of the four 


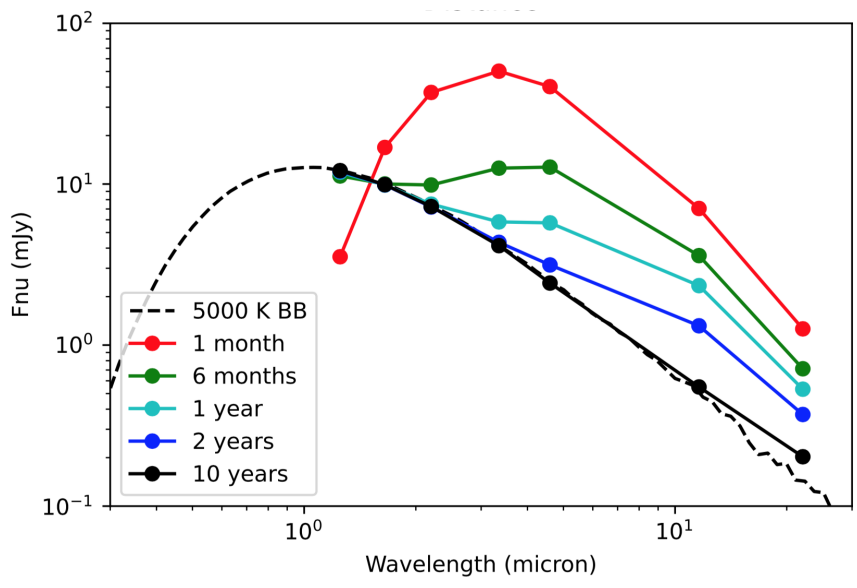

Fig. 16: Simulation of the overall SED of a 5000K RCB star which have known an episode of strong dust production of $10^{-8}$ $\mathrm{M}_{\odot}$ at $\mathrm{t}=0$. The models were made using the 3D Monte Carlo radiative transfer code named Mocassin (Ercolano et al. 2005) with a homogeneous distribution of amorphous carbon dust with a size distribution as of Cardelli et al. (1989). The star radius being of $100 \mathrm{R}_{\odot}$. The seven dots that define the dust shells'SED correspond to the 2MASS and WISE passbands. The J band luminosity of the 1 month old dust shell model is lower than the one of the photosphere blackbody due to the extinction effect. It corresponds to the characteristic RCB star photometric declines, here being of $1.2 \mathrm{mag}$ in the $\mathrm{J}$ band.

other HdC stars, three (A977, F152 and B42) have similar WISE colour to F75, but slightly bluer, indicating they may have only started a similar transition. The small $\mathrm{H}_{\alpha}$ emission line observed in the spectrum of F152 may be explained by this possible new phase associated with a strong stellar wind. The variability observed in the light curve of F152 corresponds also to the typical variabilities observed in RCB stars at maximum brightness. For the cool $\mathrm{HdC}$ star, A166, the situation appears quite different. Its [12] - [22] colour index is redder by $1.5 \mathrm{mag}$. It implies the presence of a cold $\mathrm{T}_{\text {shell }} \sim 200 \mathrm{~K}$ dust shell which would argue for a scenario where the dust production has stopped for a while, and we are seeing the dust cooling down and moving away from the star.

All the discussions above focus on the mid-IR colour that correspond mostly to one particular epoch, i.e. during the 9 months period of the WISE survey in 2010. But as was already demonstrated by Feast et al. (1997), the mid-IR fluxes of RCB stars vary in time in relation to the sudden changes of dust production from the star. We present in Fig. 16 the overall SED of an 5000K RCB star that one could observe at different epochs (between 1 month to 10 years) following a large dust production of $10^{-8}$ solar masses. We used MOCASSIN (Ercolano et al. 2005), a 3D Monte Carlo radiative transfer code, to make these models. One can see the effect of the resulting dust shell moving away from the star at $400 \mathrm{~km} . \mathrm{s}-1$ as it cools down and disperses into the interstellar environment. If an RCB star remains highly productive, a warm circumstellar dust shell is observed continually around it. Otherwise, almost no dust emission would be detected only 10 years after the production of such a high quantity of dust. We looked at the light curves of all the dLHdC stars produced by the on-going mid-IR NEOWISE survey (Mainzer et al. 2011, 2014) that observe the entire sky in the bluest two WISE passbands since 2013. We found no variations during the 8 years of observation and even similar W1 and W2 magnitudes
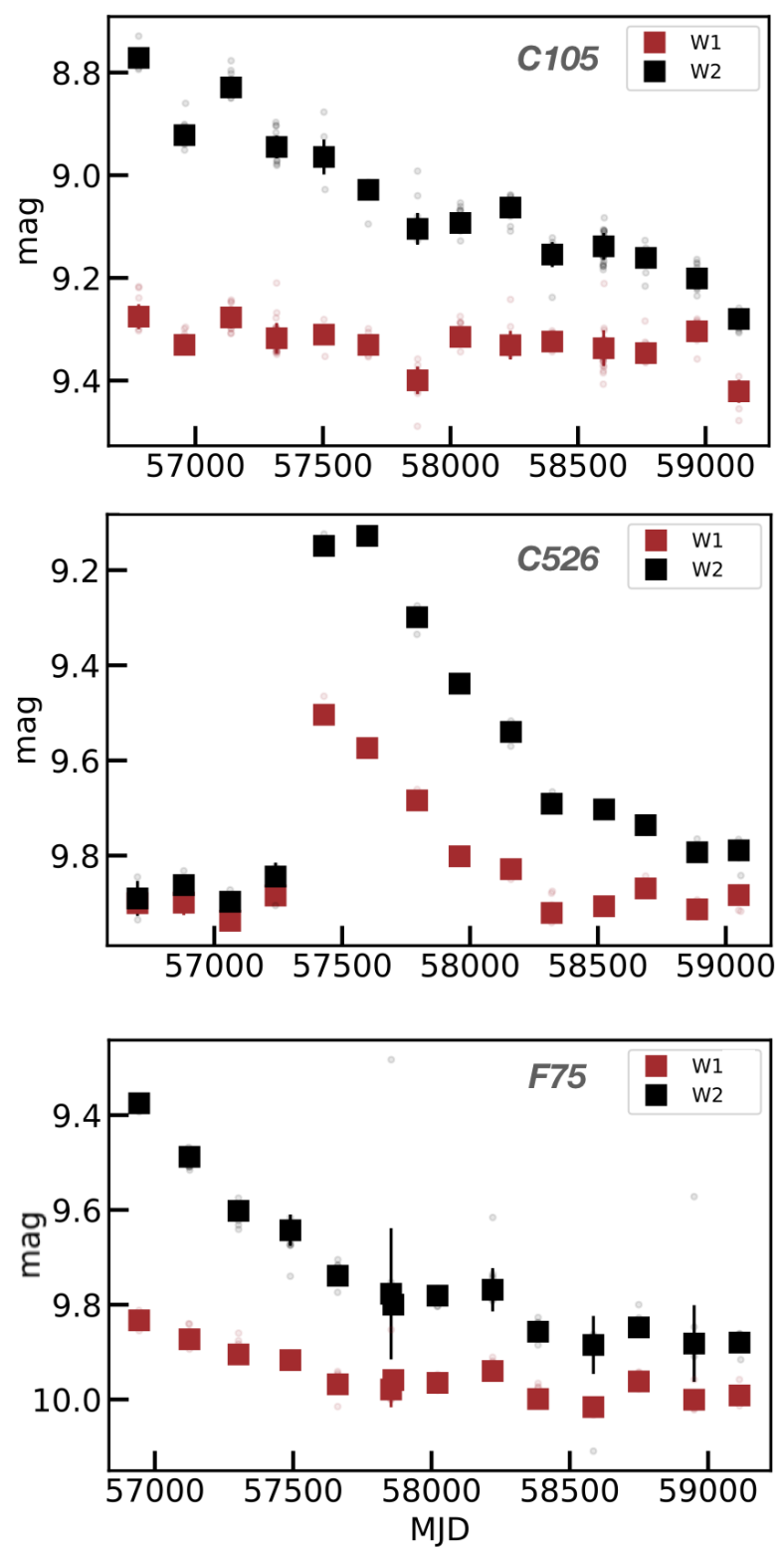

Fig. 17: Mid-IR light curve (2013-2021) from the NEOWISE survey of C105, C526 and F75. All three new warm dLHdC show Mid-IR variabilities indicating a dust production activity from these stars. The sudden increase of fluxes observed in both $\mathrm{W} 1$ and W2 bands for C526 is the signal of a rapid dust formation. The slow decreasing rate of the W2 fluxes observed in the three stars is due to the cooling down of the dust while dispersing in the circumstellar surrounding.

reported by both the WISE and the NEOWISE surveys for all except 2 dLHdC stars, F75 and C526, and C105, our new RCB star. Their respective mid-IR light curves are presented in Figure 17 In all three of them we can observe a decrease of the W2 fluxes with a total amplitude of $\sim 0.5, \sim 0.6$ and $\sim 0.5$ mag respectively for C105, C526 and F75 and a slighter one in the W1 passband of $\sim 0.1, \sim 0.4$ and $\sim 0.2 \mathrm{mag}$ respectively for these stars. It corresponds to the cooling down of the dust, that was recently newly produced near the star, as it disperses into the circumstellar environment. An emission due to a sudden dust production was even observed in 2016 for C526. In less than 200 days, the W2 flux increased by $\sim 0.8$ mag followed by a slow recovery to the value 
of the initial flux before the burst, which is also similar to the one reported by the 2010 WISE observations. The quantity of dust produced is here lower than the model presented in Fig. 16 by a factor of $\sim 20$, i.e. $\sim 5 \times 10^{-10} \mathrm{M}_{\odot}$. For $\mathrm{C} 105$, the sudden dust emission happened before 2010 as the maximum W2 (W1) flux observed in 2013 is $\sim 1.1(\sim 0.6)$ mag fainter than the one observed in 2010 . The total variation in thus of $\sim 1.6(\sim 0.5)$ mag in W2 (W1). For F75, the dust emission occurred between the two surveys, as the reported WISE magnitudes were fainter by $\sim 0.4$ $(\sim 0.3)$ mag in W2 (W1).

In conclusion, we have accumulated some evidence that shows that F75, C526 and to a lesser degree F152 are in fact producing some dust at a low rate as it does not accumulate around these stars to form a long lasting shell. These three warm stars are of the same brightness $\left(\mathrm{M}_{V}\right.$ between -4.4 and $\left.-5.0 \mathrm{mag}\right)$ as known RCB stars of the same temperature ( $7500 \mathrm{~K})$. They could be in fact typical RCB stars passing through a transition time, going in or out the RCB phase. We reached a similar conclusion for the only very cold HdC star we found, A166. A detailed discussion on that particular star is given in the following subsection.

\subsection{The specific case of $A 166$}

A166 is an HdC star that has remained apart throughout our analysis of the other dLHdC stars. It has accumulated differences in almost all observational aspects. The differences start with its location on the sky as it is a Halo star found to be at $\sim 6 \mathrm{kpc}$ away from the Sun. A166 is redder than all the newly discovered $\mathrm{HdC}$ stars revealed with a $(\mathrm{V}-\mathrm{I})_{0}$ colour index of $1.8 \mathrm{mag}$, indicating an effective temperature of about $\sim 4000 \mathrm{~K}$. It is located at a position in the colour-magnitude diagram near two known cold RCB stars (ASAS-RCB-4 and V1157 Sgr), away from the main locus of dLHdC stars (See Fig. 10). We note that its line of sight is only weakly impacted by interstellar dust reddening with $\mathrm{E}(\mathrm{B}-\mathrm{V}) \sim 0.07 \mathrm{mag}$ (similar to the two cold RCB stars).

Its cool spectrum (Fig. 5) is also uncommon. It shows no $\mathrm{CN}$ features at all, but some $\mathrm{C}_{2}$ band heads. Furthermore, along with being hydrogen-deficient, it has the curious characteristic of strong absorption lines due to s-process elements like strontium and barium (see a detailed discussion in Crawford et al. 2022).

Then, after a closer look at its entire spectral energy distribution, we found an IR excess in the reddest two WISE mid-IR photometric bands (Fig. 15). This reveals the presence of a cold dust shell with a temperature between 200 and $300 \mathrm{~K}$ (Fig. 18), not seen in any other dLHdC stars (See Sect. 4.5). The photometric variability observed in all light curves we accumulated show irregular variabilities, but one can see patterns with two long time scales of $\sim 25$ and $\sim 200$ days, and a peak-to-peak amplitude of $\sim 0.15 \mathrm{mag}$. This is not seen in any other dLHdC stars whose observed variabilities are smaller and on shorter duration.

Finally, Karambelkar et al. (2022) reported the possible detection of blue shifted He I lines in A166's IR spectrum. This is an attribute seen usually in RCB stars as it is due to a dust-driven wind (Clayton et al. 2013). Again, no other dLHdC stars show a similar pattern. Moreover, the Karambelkar et al. (2022) measurement of the oxygen 16 to 18 isotopic ratio, indicates that its enrichment in the oxygen 18 isotope is not as large as they observed for dLHdC stars, but corresponds more to ratios found in RCB stars.

In conclusion, we think that A166 is also an HdC star that is in a transition time as we are not seeing a typical warm circumstellar dust shell, but a cold one that is moving away from the star. If we consider a dust velocity of $400 \mathrm{~km} . \mathrm{s}^{-1}$, the produc-

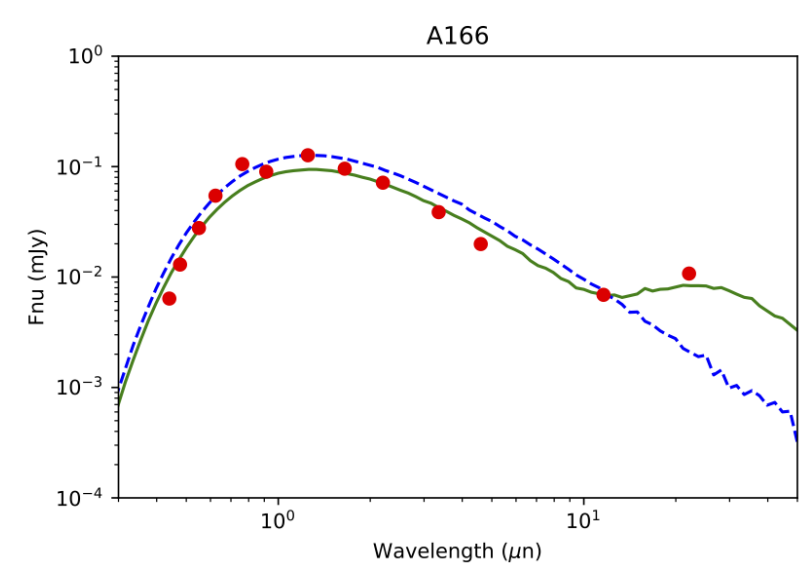

Fig. 18: Spectral Energy distribution of A166. The red dots are the photometry. The blue dashed line is a $4000 \mathrm{~K}$ black body curve. The green line adds $1.10^{-8}$ solar masses of amorphous carbon dust with a typical Cardelli et al. (1989) size distribution at a distance of $6.410^{15} \mathrm{~cm}(\sim 430 \mathrm{AU})$. It would take 5 years for the dust to reach that distance if its velocity is $400 \mathrm{~km} . \mathrm{s}^{-1}$.

tion level needed to sustain a warm dust shell must have stopped about 5 years before the 2010 WISE survey. As no noticeable mid-IR luminosity variations are observed also in its NEOWISE lightcurve between 2013 and 2021, no such massive dust production occurred during that time. We are still seeing possible signs of a dust-driven wind as observed in RCB stars, but this wind is not accompanied with large photometric declines, so A166 may be in a moderate dust production phase. These on and off phases in dust production are already seen in few known RCB stars as they are indirectly indicated by the presence of multiple circumstellar dust shells around them (see Sect. 4.5). From its position in the HR diagram, A166 could be a newly formed HdC stars that have recently started its path on the evolution track, and thus is starting its dust production phase corresponding to RCB stars.

\subsection{How many Galactic HdC stars exist out there?}

Based on their results of the first all-sky search for RCB stars using their mid-IR emissions, Tisserand et al. (2020) projected that the total number of Galactic HdC stars (i.e. the sum of RCB and dLHdC stars) should reasonably be between 300 and 500 . They also discussed a long list of uncertainties that could affect those numbers. It was mostly due to the variety of circumstellar environment observed around HdC stars, from dustless to very enshrouded, with even in some cases, the presence of multiple shells. In the case of no dust seen around HdC stars, they used the only $5 \mathrm{dLHdC}$ stars known at the time and compared that number to the number of bright RCB stars known before the modern monitoring surveys, and estimated that no more than 60 should exist in the Galaxy. Well, that number should now be revised upwards, and consequently the total number of Galactic HdC stars.

RCB stars and dLHdC stars seem to share the same Galactic distribution (Sect.4.1). To evaluate the number ratio between these two populations, we should consider only the sky area located towards the Bulge (i.e. $|1|<45 \mathrm{deg}$ and $|\mathrm{b}|<15 \mathrm{deg}$ ) in region affected by interstellar reddening $\mathrm{E}(\mathrm{B}-\mathrm{V})$ lower than $1.0 \mathrm{mag}$, as calculated by Schlafly \& Finkbeiner (2011). That corresponds to the sky area where we completed our observation of all our dLHdC candidates. There, 53 RCB stars are known, while we 
found $21 \mathrm{dLHdC}$ stars on top of the 3 known nearby ones that are also located towards that region. The ratio $\mathrm{R}_{H d C}$ in number of RCB to dLHdC stars is thus already close to 2, but we did not take into account the following three effects: 1) our search was not designed originally to find warm dLHdC stars (Sect. 3), 2) if the brightness of dLHdC stars drop suddenly for temperature below $5000 \mathrm{~K}$, there is a possibly of a selection effect towards these cool temperatures and 3) the absolute magnitude of dLHdC stars is fainter by $\sim 1.5 \mathrm{mag}$ than the one of RCB stars which could imply that we did not probe with the same detection efficiency the same volume to search for both populations.

It is already not unreasonable to imagine that the ratio $\mathrm{R}_{H d C}$ could tend to unity simply by adjusting for the first two effects, but we recognise that this a clear unknown. For the third one, we think the impact is certainly not negligible. We have shown that HdC stars have a Bulge-like distribution and we succeeded to find dLHdC stars up to $11 \mathrm{kpc}$ in the direction of the Galactic Bulge. Consequently we succeeded to probe efficiently a large fraction of the bulk of the Galactic Bulge towards the sky area surveyed. The magnitude limit reached by our survey (that is constrained by our initial star list selection with $\mathrm{G}<15$ mag and the cut on $\mathrm{M}_{G}$ afterwards, see Fig. 2 right) is faint enough to claim that we reached a detection efficiency close to values achieved by RCB stars surveys towards sky area impacted by interstellar reddening lower than $\mathrm{E}(\mathrm{B}-\mathrm{V})<0.7 \mathrm{mag}$. Above that value, our detection efficiency should decrease significantly for Bulge dLHdC stars fainter than $\mathrm{M}_{V}>-4$ mag. Furthermore, to select our initial GAIA eDR3 star list, we applied a strict cut on the parallax SNR (i.e., >3 sigma). This arbitrary choice has for direct consequence to decrease our detection efficiency as a function of distance (Luri et al. 2018). The effect is attenuated by the brightness of the sample of stars selected $(\mathrm{G}<15 \mathrm{mag})$, but we should definitely expect to be less efficient of finding dLHdC stars at the distance of the Bulge than in front of it (i.e., $\sim 6 \mathrm{kpc}$ ). We did not quantified the overall accumulated impact of the effects discussed above but this go towards a lower ratio $\mathrm{R}_{H d C}$ value.

Overall, the initial estimate from Tisserand et al. (2020) on the total number of Galactic HdC stars should be revised upwards as they used a high value of 6 for $\mathrm{R}_{H d C}$. That ratio should in fact reasonably range between 0.5 and 2 , which would then correspond to a total number of Galactic HdC stars being between 350 and 1250 when we take in account the pessimistic and optimistic scenarios described by Tisserand et al. (2020) in relation to the total number of Galactic RCB stars. RCB stars were the tip of the iceberg in the world of HdC stars. We should now recognise that $\mathrm{dLHdC}$ stars could be in similar numbers to RCB stars or even outnumber them.

\section{Summary}

After the release of the GAIA eDR3 dataset (Gaia Collaboration et al. 2021) which contains the full astrometric solution for nearly 1.5 billion sources between $3<\mathrm{G}<21$ magnitudes, we selected about $720 \mathrm{dLHdC}$ star candidates using also the near-IR photometry from the 2MASS survey in sky areas affected by interstellar reddening $\mathrm{E}(\mathrm{B}-\mathrm{V})$ lower than $1.0 \mathrm{mag}$. We defined our pragmatic selection cuts based only on the four bright dLHdC stars known at the time to optimise our rejection rate while keeping open the possibility to find new dLHdC stars over a wider ranges of temperature and brightness. We followed up spectroscopically $\sim 70 \%$ of all our targets, the remaining ones being either unreachable with our telescope during our 7 months observation campaign, or of very low priority as they are suspected of being blends with nearby objects. We discovered 27 new dLHdC stars and 1 new RCB star. We also had the fortunate surprise to reveal 2 new EHe stars, A208 and A798.

We have increased by 6-fold the number of known dLHdC stars, which allows us to better study this particular population of $\mathrm{HdC}$ stars and thus to characterise them in relation to their dusty counterpart, the RCB stars. First, we found - surprisingly - that their hydrogen-deficiency is generally less pronounced than usually seen in the atmosphere of RCB stars, as we detected traces of weak $\mathrm{H}_{\alpha}$ absorption lines in 19 of them $(\sim 63 \%)$. This was seen in only one of the four $\mathrm{dLHdC}$ star previously known, i.e. HD 148839. Second, most of the new dLHdC stars show strong $\mathrm{C}_{2}$ features but only weak $\mathrm{CN}$ bands, indicating that their atmospheres have a lower nitrogen abundance than the RCB stars.

Third, we found that a few of the new stars are located in the Galactic Halo (A166, F152, F75, C20 and HE 1015-2050), but most seem to have positions and geometric distances that support a Bulge-like distribution, similarly to RCB stars.

Fourth, our study of the WISE mid-IR photometry for all new $\mathrm{HdC}$ stars confirm that a large majority of them are indeed dustless, and that one, C105, is a new RCB star surrounded by a typical warm circumstellar shell of $\sim 500 \mathrm{~K}$. Furthermore, a few of them show some sign of a moderate dust production. This is the case for F75, F152, C526 and A166. They appear to be in a transition time, going into or going out of the RCB phase, and may therefore not be part of this dustless population of $\mathrm{HdC}$ stars. This is supported principally by their WISE photometry that seems to indicate the presence of a thin layer of warm circumstellar dust shell for the first two, and a cold distant shell for the later, A166. A166 could be a supergiant HdC star that has started its path on the evolution track not long ago, and is now starting up a dust production phase like an RCB star. Evidence of a moderate amount of dust production for C526 comes from a burst lasting 5 years monitored by the NEOWISE mid-IR survey. Small photometric declines ( $\sim 0.4 \mathrm{mag})$ were even observed in the visual light curves of F75, as well as some emission lines in its spectrum, like we did during our survey for F152.

Fifth, we studied the light curves of the new dLHdC stars and found that they present either no detectable variability at the photometric resolution of the ASAS-SN monitoring survey, or if they do, present some irregular variabilities of weak peakto-peak amplitude (typically $\sim 0.1 \mathrm{mag}$ ) and of short timescale ( $\sim 10$ days). This is in clear contrast with RCB stars that show variabilities at maximum brightness of the order of 0.3-0.4 mag in total amplitude and with typical timescales between 10 to 60 days.

Sixth, astonishingly, the population of dLHdC stars is clearly intrinsically fainter than the RCB stars population. In a reconstructed $\mathrm{M}_{V}$ vs $(\mathrm{V}-\mathrm{I})_{0}$ colour magnitude diagram, we found that within the following colour range, $0.4<(\mathrm{V}-\mathrm{I})_{0}<1.0 \mathrm{mag}$, dL$\mathrm{HdC}$ stars are distributed with a median absolute magnitude $\mathrm{M}_{V}^{d L H d C} \sim-3$ mag while Galactic RCB stars have $\mathrm{M}_{V}^{R C B}$ of -4.5 mag. We can even observe that the brightness of the entire population of HdC stars is distributed over 3 magnitudes for the same temperature range. This is in complete contradiction to the first view we had from simply using the Magellanic RCB stars (Alcock et al. 2001, Tisserand et al. 2009). We also did not find any dLHdC stars cooler than $\sim 5000 \mathrm{~K}$, if we consider A166 to be an RCB star. We do not have a strong argument for a selection effect that could explain such a missing sample of stars, and one should consider that it is a physical effect related to the evolution of this group of stars. We will focus on searching for cold dLHdC stars to test this hypothesis.

Overall, apart from the amount of circumstellar dust surrounding them, our survey has revealed new differences between 
both populations of HdC stars: hydrogen and nitrogen abundances, photometric variability, absolute magnitude and temperature range. These need to be added on top of the already suspected one which is the lower ${ }^{16} \mathrm{O} /{ }^{18} \mathrm{O}$ ratio observed in the atmosphere of dLHdC stars Clayton et al. (2007); GarcíaHernández et al. (2009) and Karambelkar et al. (2022). HdC stars' atmospheres, including RCB stars, are rich in the oxygen 18 isotope (Clayton et al.2007), but the dustless ones appear to be the richest.

In the framework of the double degenerate scenario, which is the favourite scenario to explain the origin of HdC stars (Webbink 1984, Jeffery et al. 2011, Clayton 2012), the large range in absolute magnitude observed (up to 3 mags) could be explained by the fact that we are seeing a series of evolutionary sequences of white dwarf mergers with a large range of initial total mass. We presented the result of a population synthesis simulation whose outcome is a plausible distribution of this initial total mass. It ranges between 0.6 and $1.05 \mathrm{M}_{\odot}$ with a clear bimodal structure. The massive end of the mass distribution being formed mostly by the merger of an 'hybrid' COHe WD with a CO WD. The smaller the total mass, the fainter the resulting supergiant HdC star is after its evolution to warmer temperatures. Such evolutionary sequences were simulated by Saio \& Jeffery (2002). They even show that the initial phase of HdC stars, just after the merger, starts at warmer temperature for smaller total masses. This effect could explain our missing population of cold dLHdC stars. Furthermore, a noticeable difference is observed between the shape of the distribution of absolute magnitudes between the Galactic RCB stars and the Magellanic ones. They both show similar maximum luminosity at warmer temperature but a difference up to $\sim 1.5 \mathrm{mag}$ on the cooler side. Magellanic cold RCB stars being brighter. This could be due to a physical difference in the original stellar population in metallicity. Indeed, Romero et al. (2015) have shown that metal-rich progenitors result in less massive WD remnants due to an enhancement of the mass-loss rates associated with high metallicity values. However, to match our observations, Saio \& Jeffery (2002) simulations show that the resulting Magellanic WDs systems need to be of a similar total mass than the Galactic ones. The difference being only on the WDs mass ratio. This will need further investigation. We are also observing an envelop of maximum brightness versus temperature in the distribution of $\mathrm{HdC}$ stars in the $\mathrm{M}_{V}$ vs (V-I) $)_{0}$ colour-magnitude diagram. We interpret this as the evolutionary sequence of the maximum total white dwarf mass possible to create an HdC star. Above that mass threshold, the WD mergers may result in supernovae (Sim et al. 2010; Ruiter et al.2011). Finally, we note that if dLHdC stars are indeed the result of WD mergers of lower total mass than the ones that create RCB stars, the physical process needed to create dust from atmospheric convection is highly dependent on this initial total mass.

As a whole, our observations satisfy projections made for the double degenerate scenario. We have presented new and strong arguments that support this formation model for HdC stars.

We now have several pieces of evidence that show that an underlying population of real "long-term" dustless HdC stars exists and that they are characterised by the group of dLHdC stars of lower luminosity with $\mathrm{M}_{V} \sim-3 \mathrm{mag}$, as no RCB star is known in that luminosity and temperature range. This population of dLHdC stars is not simply a phase within the evolution of an RCB star to its subsequent stage, an EHe star, as previously thought. We think they are in fact a different population of HdC stars that evolve on their own and that are potentially producing no dust during their lifetimes or at such a low rate that no circumstellar dust shell has the time to ever form. We will need to define the contour of this underlying population. Visual to mid-IR photometric monitoring could help to find those that are in a transition time into or out of the RCB phase, like we have seen with F75, F152, C526 and A166. The new sample of dLHdC stars we discovered opens a new window in the quest to decipher the origin of HdC stars. RCB stars are the tip of the iceberg in that world of stars. There could exist as many dLHdC stars as RCB stars in our Galaxy, if not more. Overall, we estimate the total number of Galactic HdC stars to be between 350 and 1250 .

We should be able to further constrain this number with upcoming surveys that will focus on searching for the warmest and coolest dLHdC stars. Our fortunate discovery of two EHe stars has also led to some ideas on how to design a dedicated search for these rare helium-rich blue supergiant stars. Finally, we underline the importance of future detailed abundance analysis of this population of dLHdC stars similar to those already made for RCB stars (Asplund et al. 2000, Hema et al. 2012, 2017, Pandey et al. 2021) to recognise possible differences between both populations caused by their distinct original white dwarf systems in age, mass and mass ratio.

Acknowledgements. PT is profoundly grateful for the long unconditional support and love from his father, Claude Tisserand (1945-2020), and this paper is dedicated to his memory.

He personally thanks Tony Martin-Jones for his usual highly careful reading and comment. PT acknowledge also financial support from "Programme National de Physique Stellaire" (PNPS) of CNRS/INSU, France. AJR is funded through the Australian Research Council under award number FT170100243. We also thank the team located at Siding Spring Observatory that keeps the $2.3 \mathrm{~m}$ telescope and its instruments in good shape, as well as the engineer, computer and technician teams located at Mount Stromlo Observatory that have facilitated the observations. Palomar Gattini-IR (PGIR) is generously funded by Caltech, the Australian National University, the Mt Cuba Foundation, the Heising Simons Foundation and the Binational Science Foundation. PGIR is a collaborative project among Caltech, the Australian National University, the University of New South Wales, Columbia University and the Weizmann Institute of Science. MMK acknowledges generous support from the David and Lucille Packard Foundation. MMK and EO acknowledge the US-Israel Bi-national Science Foundation Grant 2016227. MMK and JLS acknowledge the Heising-Simons foundation for support via a Scialog fellowship of the Research Corporation. MMK and AMM acknowledge the Mt Cuba foundation. J. Soon is supported by an Australian Government Research Training Program (RTP) Scholarship. This work has made use of data from the European Space Agency (ESA) mission Gaia (https://www.cosmos.esa.int/gaia), processed by the Gaia Data Processing and Analysis Consortium (DPAC, https://www.cosmos.esa.int/web/ gaia/dpac/consortium). Funding for the DPAC has been provided by national institutions, in particular the institutions participating in the Gaia Multilateral Agreement. This publication makes use of data products from the Two Micron All Sky Survey, which is a joint project of the University of Massachusetts and the Infrared Processing and Analysis Center/California Institute of Technology, funded by the National Aeronautics and Space Administration and the National Science Foundation. This publication makes use of data products from the Wide-field Infrared Survey Explorer, which is a joint project of the University of California, Los Angeles, and the Jet Propulsion Laboratory/California Institute of Technology, funded by the National Aeronautics and Space Administration. This research has made use of the SIMBAD database, operated at CDS, Strasbourg, France. The DASCH project at Harvard is grateful for partial support from NSF grants AST-0407380, AST-0909073, and AST-1313370.

\section{References}

Alcock, C., Allsman, R. A., Alves, D. R., et al. 2001, ApJ, 554, 298 Alcock, C., Allsman, R. A., Alves, D. R., et al. 1996, ApJ, 470, 583 Alksnis, A., Balklavs, A., Dzervitis, U., et al. 2001, Baltic Astronomy, 10, 1 Andrae, R., Fouesneau, M., Creevey, O., et al. 2018, A\&A, 616, A8

Asplund, M., Gustafsson, B., Lambert, D. L., \& Rao, N. K. 2000, A\&A, 353, 287

Bailer-Jones, C. A. L., Rybizki, J., Fouesneau, M., Demleitner, M., \& Andrae, R. 2021, AJ, 161, 147

Belczynski, K., Kalogera, V., Rasio, F. A., et al. 2008, ApJS, 174, 223

Bidelman, W. P. 1953, ApJ, 117, 25 
Buder, S., Sharma, S., Kos, J., et al. 2021, MNRAS, 506, 150

Burggraaff, O., Talens, G. J. J., Spronck, J., et al. 2018, A\&A, 617, A32

Cardelli, J. A., Clayton, G. C., \& Mathis, J. S. 1989, ApJ, 345, 245

Childress, M. J., Vogt, F. P. A., Nielsen, J., \& Sharp, R. G. 2014, Ap\&SS, 349 617

Clayton, G. C. 1996, PASP, 108, 225

Clayton, G. C. 2012, Journal of the American Association of Variable Star Observers (JAAVSO), 40, 539

Clayton, G. C., Geballe, T. R., Herwig, F., Fryer, C., \& Asplund, M. 2007, ApJ, 662,1220

Clayton, G. C., Geballe, T. R., \& Zhang, W. 2013, AJ, 146, 23

COROT Team. 2016, VizieR Online Data Catalog, B/corot

Crawford, C. L., Tisserand, P., Clayton, G. C., \& Munson, B. 2022, A\&A, Submitted

Crocker, R. M., Ruiter, A. J., Seitenzahl, I. R., et al. 2017, Nature Astronomy, 1, 0135

Cutri R.M., Wright E.L., C. T. F. J. E. P. G. C. K. J. M. F. M. H. W. S. F.-A. S Y. L. B. D. H. M. J. T. L. S. L. D. R. M. S. S. T. C.-W. L. F. H. G. M. A. G. D. G. A. H. D. M. K. P. D. S. M. B. R. P. M. W. M. 2013, VizieR Online Data Catalog: AllWISE Data Release

De, K., Hankins, M. J., Kasliwal, M. M., et al. 2020, PASP, 132, 025001

Dopita, M., Hart, J., McGregor, P., et al. 2007, Ap\&SS, 310, 255

Drake, A. J., Djorgovski, S. G., Mahabal, A., et al. 2012, in New Horizons in Time Domain Astronomy, ed. E. Griffin, R. Hanisch, \& R. Seaman, Vol. 285, 306-308

Droege, T. F., Richmond, M. W., Sallman, M. P., \& Creager, R. P. 2006, PASP, 118,1666

Ducati, J. R. 2002, VizieR Online Data Catalog

Ercolano, B., Barlow, M. J., \& Storey, P. J. 2005, MNRAS, 362, 1038

Feast, M. W., Carter, B. S., Roberts, G., Marang, F., \& Catchpole, R. M. 1997, MNRAS, 285, 317

Fleming, M. 1892, Astronomy and Astro-Physics (formerly The Sidereal Messenger), 11,765

Gaia Collaboration, Babusiaux, C., van Leeuwen, F., et al. 2018, A\&A, 616, A10

Gaia Collaboration, Brown, A. G. A., Vallenari, A., et al. 2021, A\&A, 649, Al

Gaia Collaboration, Prusti, T., de Bruijne, J. H. J., et al. 2016, A\&A, 595, A1

García-Hernández, D. A., Hinkle, K. H., Lambert, D. L., \& Eriksson, K. 2009, ApJ, 696, 1733

García-Hernández, D. A., Rao, N. K., \& Lambert, D. L. 2011, ApJ, 739, 37

Goswami, A. \& Aoki, W. 2013, ApJ, 763, L37

Goswami, A., Karinkuzhi, D., \& Shantikumar, N. S. 2010, ApJ, 723, L238

Green, G. M., Schlafly, E., Zucker, C., Speagle, J. S., \& Finkbeiner, D. 2019, ApJ, 887, 93

Hartman, J. D., Bakos, G., Stanek, K. Z., \& Noyes, R. W. 2004, AJ, 128, 1761

Heinze, A. N., Tonry, J. L., Denneau, L., et al. 2019, VizieR Online Data Catalog, $\mathrm{J} / \mathrm{AJ} / 156 / 241$

Hema, B. P., Pandey, G., Kamath, D., et al. 2017, PASP, 129, 104202

Hema, B. P., Pandey, G., \& Lambert, D. L. 2012, ApJ, 747, 102

Jayasinghe, T. Kochanek, C. S., Stanek, K. Z., et al. 2018, MNRAS, 477, 3145

Jeffery, C. S. 2008, in Astronomical Society of the Pacific Conference Series, Vol. 391, Hydrogen-Deficient Stars, ed. A. Werner \& T. Rauch, 53

Jeffery, C. S., Karakas, A. I., \& Saio, H. 2011, MNRAS, 414, 3599

Karakas, A. I., Ruiter, A. J., \& Hampel, M. 2015, ApJ, 809, 184

Karambelkar, V. R., Kasliwal, M. M., Tisserand, P., et al. 2021, ApJ, 910, 132

Karambelkar et al., V. R. 2022, A\&A, Submitted

Kilkenny, D. \& Marang, F. 1989, MNRAS, 238, 1P

Kipper, T. 2002, Baltic Astronomy, 11, 249

Kochanek, C. S., Shappee, B. J., Stanek, K. Z., et al. 2017, PASP, 129, 104502

Lawson, W. A. 1992, Monthly Notices of the Royal Astronomical Society, 258, $33 \mathrm{P}$

Lawson, W. A. \& Cottrell, P. L. 1989, MNRAS, 240, 689

Lindegren, L., Bastian, U., Biermann, M., et al. 2021a, A\&A, 649, A4

Lindegren, L., Klioner, S. A., Hernández, J., et al. 2021b, A\&A, 649, A2

Luri, X., Brown, A. G. A., Sarro, L. M., et al. 2018, A\&A, 616, A9

Lynas-Gray, A. E., Walker, H. J., Hill, P. W., \& Kaufmann, J. P. 1981, A\&AS, 44,349

Mainzer, A., Bauer, J., Cutri, R. M., et al. 2014, ApJ, 792, 30

Mainzer, A., Bauer, J., Grav, T., et al. 2011, ApJ, 731, 53

Malhan, K., Ibata, R. A., \& Martin, N. F. 2018, MNRAS, 481, 3442

Montiel, E. J., Clayton, G. C., Marcello, D. C., \& Lockman, F. J. 2015, AJ, 150, 14

Montiel, E. J., Clayton, G. C., Sugerman, B. E. K., et al. 2018, AJ, 156, 148

Moore, A. M. \& Kasliwal, M. M. 2019, Nature Astronomy, 3, 109

Morel, M. \& Magnenat, P. 1978, A\&AS, 34, 477

Nelemans, G., Yungelson, L. R., Portegies Zwart, S. F., \& Verbunt, F. 2001, A\&A, 365, 491

Oelkers, R. J., Rodriguez, J. E., Stassun, K. G., et al. 2018, AJ, 155, 39

Pakmor, R., Zenati, Y., Perets, H. B., \& Toonen, S. 2021, MNRAS, 503, 4734

Pandey, G., Hema, B. P., \& Reddy, A. B. S. 2021, ApJ, 921, 52
Pandey, G., Kameswara Rao, N., Lambert, D. L., Jeffery, C. S., \& Asplund, M. 2001, MNRAS, 324, 937

Parkhurst, J. A. 1912, ApJ, 35, 125

Pickering, E. C. \& Fleming, W. P. 1896, ApJ, 4, 142

Pojmanski, G. 1997, Acta Astronomica, 47, 467

Rao, N. K. \& Lambert, D. L. 2008, MNRAS, 384, 477

Rimoldini, L., Dubath, P., Süveges, M., et al. 2012, MNRAS, 427, 2917

Romero, A. D., Campos, F., \& Kepler, S. O. 2015, MNRAS, 450, 3708

Ruiter, A. J., Belczynski, K., \& Fryer, C. 2009, ApJ, 699, 2026

Ruiter, A. J., Belczynski, K., Sim, S. A., et al. 2011, MNRAS, 417, 408

Ruiter, A. J., Belczynski, K., Sim, S. A., Seitenzahl, I. R., \& Kwiatkowski, D.

2014, MNRAS, 440, L101

Saio, H. \& Jeffery, C. S. 2002, MNRAS, 333, 121

Schlafly, E. F. \& Finkbeiner, D. P. 2011, ApJ, 737, 103

Shappee, B. J., Prieto, J. L., Grupe, D., et al. 2014, ApJ, 788, 48

Shields, J. V., Jayasinghe, T., Stanek, K. Z., et al. 2019, MNRAS, 483, 4470

Sim, S. A., Röpke, F. K., Hillebrandt, W., et al. 2010, ApJ, 714, L52

Skrutskie, M. F., Cutri, R. M., Stiening, R., et al. 2006, AJ, 131, 1163

Stephenson, C. B. 1973, Publications of the Warner \& Swasey Observatory

Stock, J. \& Wroblewski, H. 1972, Publications of the Department of Astronomy

University of Chile, 2, 59

Tisserand, P. 2012, A\&A, 539, A51

Tisserand, P., Clayton, G. C., Bessell, M. S., et al. 2020, A\&A, 635, A14

Tisserand, P., Clayton, G. C., Welch, D. L., et al. 2013, A\&A, 551, A77

Tisserand, P., Marquette, J. B., Beaulieu, J. P., et al. 2004, A\&A, 424, 245

Tisserand, P., Marquette, J. B., Wood, P. R., et al. 2008, A\&A, 481, 673

Tisserand, P., Wood, P. R., Marquette, J. B., et al. 2009, A\&A, 501, 985

Tisserand, P., Wyrzykowski, L., Wood, P. R., et al. 2011, A\&A, 529, A118

Tonry, J. L., Denneau, L., Flewelling, H., et al. 2018, ApJ, 867, 105

Tutukov, A. \& Yungelson, L. 1996, MNRAS, 280, 1035

Udalski, A. 2008, Acta Astronomica, 58, 187

Walker, H. J. 1985, A\&A, 152, 58

Warner, B. 1963, MNRAS, 126, 61

Warner, B. 1967, MNRAS, 137, 119

Webbink, R. F. 1984, ApJ, 277, 355

Wenger, M., Ochsenbein, F., Egret, D., et al. 2000, A\&AS, 143, 9

Wright, E. L., Eisenhardt, P. R. M., Mainzer, A. K., et al. 2010, AJ, 140, 1868

Zaniewski, A., Clayton, G. C., Welch, D. L., et al. 2005, AJ, 130, 2293

Zenati, Y., Toonen, S., \& Perets, H. B. 2019, MNRAS, 482, 1135 